\title{
Consensus Control for Reactive Power Sharing Using an Adaptive Virtual Impedance Approach
}

\author{
Ahmed S. Alsafran *(D) and Malcolm W. Daniels \\ Electrical and Computer Engineering Department, University of Dayton, Dayton, OH 45469, USA; \\ mdaniels1@udayton.edu \\ * Correspondence: alsafrana1@udayton.edu
}

Received: 20 January 2020; Accepted: 15 April 2020; Published: 18 April 2020

\begin{abstract}
Reactive power sharing among distributed generators (DGs) in islanded microgrids (MGs) presents control challenges, particularly in the mismatched feeder line condition. Improved droop control methods independently struggle to resolve this issue and centralized secondary control methods exhibit a high risk of collapse for the entire MG system under any failure in the central control. Distributed secondary control methods have been recently proposed to mitigate the reactive power error evident in the presence of mismatched feeder lines. This paper details a mathematical model of an adaptive virtual impedance control that is based on both leaderless and leader-followers consensus controls with a novel triangle mesh communication topology to ensure accurate active and reactive power sharing. The approach balances an enhanced rate of convergence with the anticipated implementation cost. A MATLAB/Simulink model with six DG units validates the proposed control performance under three different communication structures: namely, ring, complete, and triangle mesh topologies. The results suggest that leaderless consensus control is a reliable option with large DG systems, while the leader-followers consensus control is suitable for the small systems. The triangle mesh communication topology provides a compromise approach balancing the rate of convergence and the expected cost. The extensibility and scalability are advantages of this topology over the alternate ring and complete topologies.
\end{abstract}

Keywords: adaptive virtual impedance; hierarchical control; islanded microgrids; leaderless consensus control; leader-followers consensus control; mismatched feeder lines; reactive power sharing; triangle mesh communication topology

\section{Introduction}

Recently, increased power demand has been one of the primary challenges associated with the power systems in many countries. Smart Grid (SG) technologies provide solutions for meeting the increased load demand; ensuring power quality, reliability, and efficiency; and, reducing the emission of carbon dioxide associated with global climate change [1]. Microgrids (MGs) are one of the dominant Smart Grid technologies that have attracted research interest in response to efforts to integrate distributed generators (DG) into the utility grid or as standalone systems [1]. Research has extensively investigated the design, operation, and control of these microgrids, particularly in the islanded mode. Numerous studies have proposed hierarchical control techniques to manage both power flow and power sharing among distributed generators (DGs) in the islanded case [2-4]. However, in the interconnected DG case, the lack of effective power sharing among DGs and inverters causes mismatched power sharing that might damage the DG sources and often leads to instability in the microgrid during transient changes in the load $[2,3,5]$.

Sharing reactive power presents a significant challenge in the case of mismatched feeder lines where feeder line impedances are different from one to another. The influence of feeder line impedance 
presents challenges to the regulation of the output voltage and current in order to achieve the desired performance. In addition, the output voltage and frequency are known to deviate from the desired reference values in the event of any disturbances Consequently, it is necessary to match the feeder line impedances and regulate the output currents properly in order to eliminate the consequences of the mismatch condition in order to achieve accurate power [6].

Sharing reactive power among DGs in an islanded microgrid using conventional or improved droop control independently under mismatched feeder lines condition has proven difficult [1-9]. Numerous researchers have proposed distributed secondary control methods for eliminating the reactive power error in the mismatched feeder line condition and mitigate the drawback of a high risk of collapse in the entire system under any failure in the centralized secondary control [10-16]. Distributed secondary control methods that are based on consensus control algorithms, such as average consensus, leaderless consensus, and leader-followers consensus control have been proposed to eliminate reactive power errors [2,3,17-26]. Additionally, Droop controls that are based on distributed consensus control methods have been proposed that achieve accurate reactive power sharing $[19,21,27]$, although the methods eliminate the reactive power errors with a relatively slow rate of convergence. A new consensus control method that ensures a fast rate of convergence is needed. Since the consensus control method also depends on the MG communication topology, a new communication topology is needed in order to enhance the rate of convergence while considering the connectivity, extensibility, scalability, and cost of the topology.

This study describes the design of a new adaptive virtual impedance secondary control that is based on a consensus control algorithm to provide accurate reactive power sharing among DGs in an islanded microgrid with mismatched feeder line impedances. The proposed control method will extend previous work in the literature to enhance the rate of convergence of reactive power sharing when considering the connectivity of the communication topology [8,28-30]. A new triangle mesh communication topology is designed that balances the rate of convergence and the system cost. The topology prevents overloading on any communication agent and reduces the risk of delays inherent in exchanging information between agents.

This paper develops the theoretical background, methodology, and simulation of the proposed control method. The model of the electrical network of an islanded microgrid and different communication topologies are discussed in detail. The paper provides a detailed mathematical the modeling of both the primary and secondary control levels of a hierarchical control that is designed for ensure accurate active and reactive power sharing across all DG units. The proposed communication topology is presented to validate this proposed topology for any future extensions relative to extensibility and scalability. An analytical study of the reactive power and stability analysis of the proposed consensus control method is discussed in detail. The model of electrical system with different communication topologies, including proposed topology, is simulated in MATLAB/Simulink to validate the proposed control.

\section{Background}

\subsection{Modeling of AC Electrical Network of a Microgrid}

In the islanded mode, an AC microgrid is a standalone system that is disconnected from the main grid that has full responsibility to manage the entire MG system to supply the demand power to the loads [3-5,31]. An islanded AC microgrid can contain a large number of DG units to meet the demand power. Here, the islanded microgrid is considered as a ring feeder distribution system developed to connect the DG units in one electric power distribution system, as shown in Figure 1 below. 


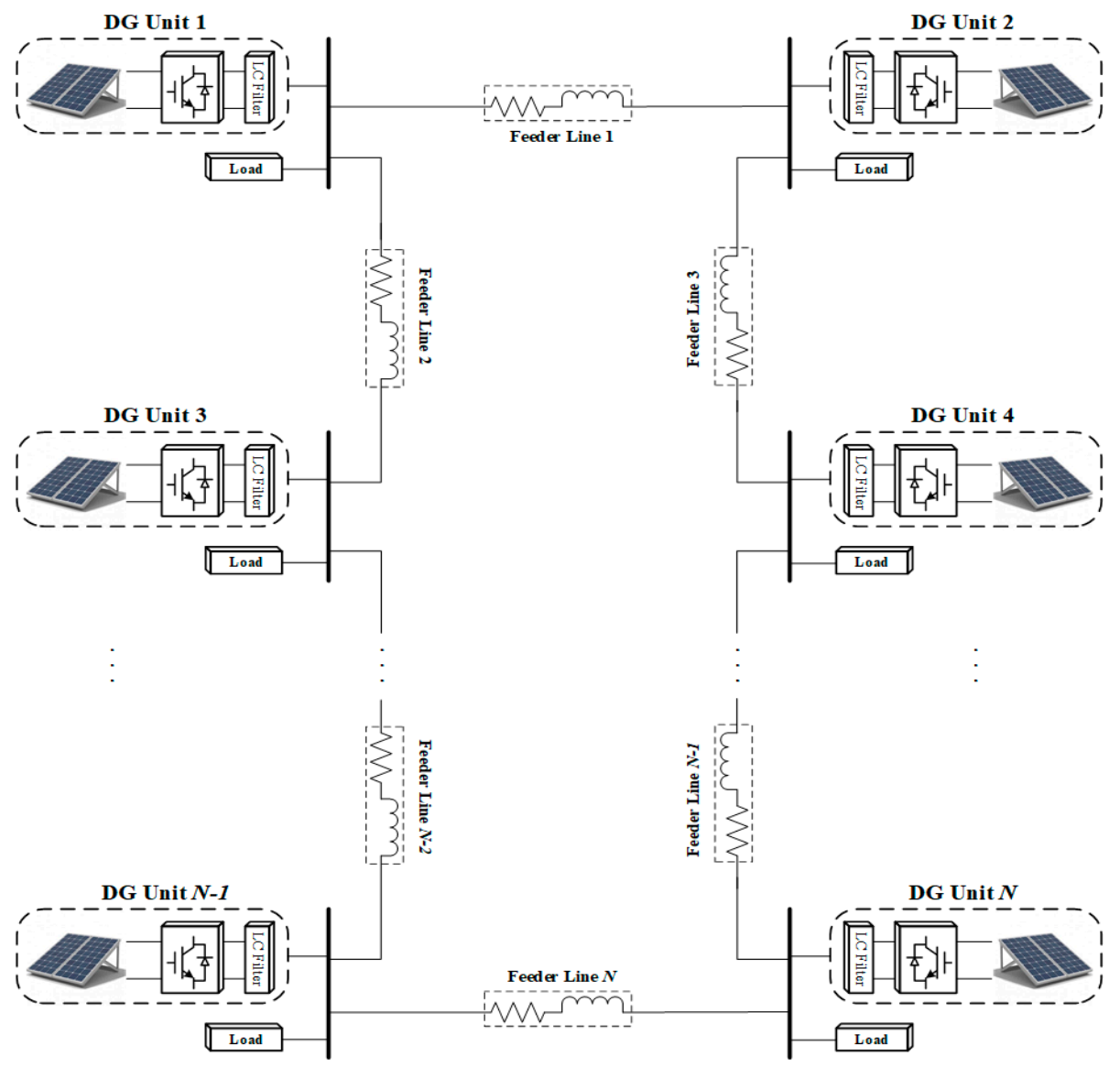

Figure 1. Modeling of an AC electrical network of islanded microgrid.

Figure 1 shows the model of an AC electrical network of microgrid that consists of a number $(N)$ DG units indexed by $i=1,2, \ldots, N$. Each DG unit is connected through feeder lines to two other DGs that can be considered to be its neighbors ( $j^{\text {th }}$ DG units).

\subsection{Modeling of the Communication Network of the Microgrid}

The communication topology is defined as the arrangement of agents and communication lines of the communication network [32]. Building a communication network is required in the secondary control level of the hierarchal control to allow for the DG units to communicate with each other. In general, communication agents do not need to have a direct communication link to all other agents in the system. Rather, the existence of some communication path to every agent via other agents is necessary to guarantee the communication topology connectivity $[19,20,32,33]$.

\subsubsection{A Graph Theory Approach}

Mathematically, the communication network of the microgrid can be represented using graph theory, an approach that permits the description of the communication infrastructure [33].

\section{Definitions:}

Definition 1. A graph $(\mathcal{G})$ consists of a collection of agents $(\mathcal{V})$ and a collection of edges $(\mathcal{E})$, that can be written as $\mathcal{G}=(\mathcal{V}, \mathcal{E})$. Each edge $e \in \mathcal{E}$ joins two nodes $u$ and $v$ at its endpoints. Given e joins $i$ and $j \in \mathcal{V}$, we can write $e=\langle i, j\rangle$. In this case, agents $u$ and $v$ are known as adjacent, and edge $e$ is known as incident with agents $u$ and $v$ respectively. Often, $\mathcal{V}$ is written as $\mathcal{V}(\mathcal{G})$ and $\mathcal{E}$ as $\mathcal{E}(\mathcal{G})$ to denote the collection of agents and edges associated with the graph $(\mathcal{G})$ respectively [32]. 
Definition 2. An undirected graph is a set or collection of agents and unordered or undirected set or collection of edges. There is no distinction between $\langle i, j\rangle$ and $\langle j, i\rangle$, they both denote that agents $u$ and $v$ are adjacent. In the other word, $e_{i j}=e_{j i}[32]$.

Definition 3. Connectivity means that each agent $(v)$ can be reached from any other agent $(w)$ via a chain of adjacent agents $(v)$ to $(w)$. Connectivity is very important to the robustness of the networks. Here, robustness means how well the network stays connected when agents or edges are removed or disconnected [32].

Figure 2 illustrates an example of a connected graph to clarify these definitions. This graph considered as an undirected graph because the edges between agents are unordered or undirected. Thus, the communication here is bidirectional and there are two paths to exchange information between agents, for example, the red dash arrow in Figure 2 indicates the short path between agent 3 and agent 6 , while the blue dot arrow indicates the long path. In this graph, the degree of the neighbor set of all agents is two, since all agents have two neighbors. Thus, this graph is a connected graph, but it is not a complete graph, since there is no direct link between all agents.

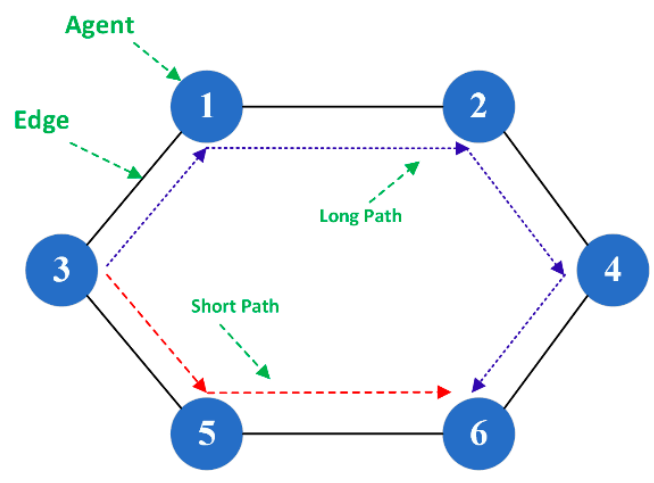

Figure 2. An example of a connected graph.

\subsubsection{Communication Topology}

The communication topology is the schematic description of how the MG communication network connects different agents [32]. Numerous communication topologies exist in the literature, such as bus, star, ring, etc. [21-24]. The importance of the communication topology here is to determine how the information flow within the network directly influences the performance of the power network.

Communication topologies can be categorized into centralized, decentralized, and distributed networks [21-24]. A centralized communication topology, where a central agent is connected to all other agents, is known to be sensitive to central agent failures that easily shut down the entire system $[21,22,24]$. In more complex decentralized communication topologies, certain distributed communication topology has recently gained popularity. In this case, since every agent makes its own decision based on a consensus protocol [21-24], communication link failures do not have a significant impact on the entire system. If a neighboring communication links fails, the agent can communicate to another neighbor to share or exchange information [24].

A popular example of distributed communication is the ring communication topology where each agent connects to exactly two neighboring agents, as shown in Figure 3. Ring communication is the simplest distributed topology and the most inexpensive one, since it only requires one communication link for each agent [21]. Here, adding or deleting agents requires interrupting the network activity and studies have shown that a long time is required to reach a consensus between agents in the network when compared to other distributed communication topologies [21,24]. 


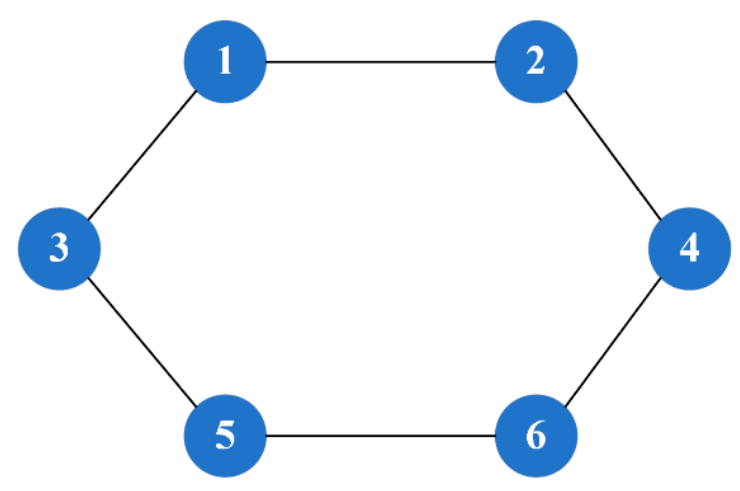

Figure 3. An example of six distributed generator (DG) units connected based on ring communication topology.

Figure 4 illustrates another example of a distributed communication topology is the complete communication topology. Here, every agent communicates with all other agents to reach a consensus decision. Thus, all of the agents act as central agents ensuring a robust system that exhibits the fastest coverages to the consensus region when compared to other distributed topologies [21,24]. This structure requires a substantial number of communication links that increase the cost of the communication infrastructure [21]. Additionally, expanding this topology is highly expensive, especially in large systems [21].

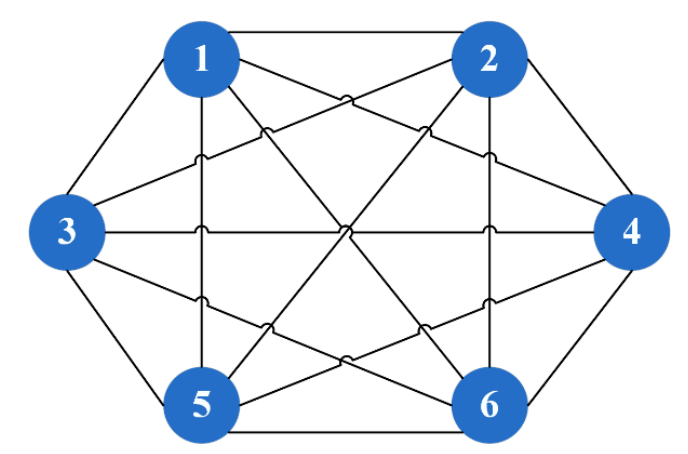

Figure 4. An example of six DG units connected based on complete communication topology.

\section{Assumptions and Methods}

\subsection{Proposed Communication Topology}

A consideration of the ring and complete communication topologies' performance characteristics suggests a need to build a new topology that balances the rate of convergence and the system cost, which also takes into consideration the network's extensibility, scalability, and fault tolerance. Here, a new communication topology is proposed to achieve this goal and it is termed a triangle mesh communication topology.

Defining terms according to definitions in $[24,25]$, the degree of all agents, which is the number of agents incident with an agent, for this proposed control (a measure of an agent's connectivity to the network) is not the same, unlike the ring topology (degree of 2) and the complete topology (degree of $N-1$ ). Here, the highest degree of any agent is four for agents located at the center of the communication graph. The other terminal agents have a degree of two or three. For the example shown in Figure 5, agents 3 and 4 have a degree of four, and agents 1 and 6 and agents 2 and 5 have a degree of three and two, respectively. This proposed topology is easy to expand and expanding causes extensive growth of centralized agents that have a degree of four. In addition, an extension of the system does not require any interruption to the network activity. 


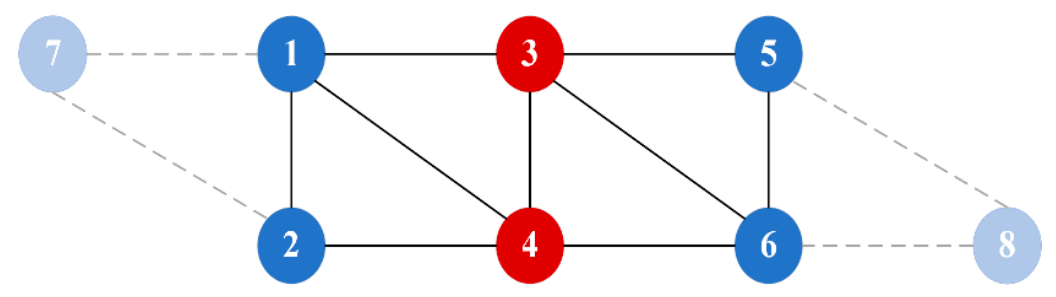

Figure 5. An example of six DG units connected based on Triangle mesh communication topology.

With reference to Figure 5, above, two new agents (agents 7 and 8 ) are added to the communication topology, with two communication links that are necessary to connect each new agent into the communication topology Adding a new agent causes changes in the degree of non-maximum agents in the network, and generates a new agent with a degree 4 . Thus, agents 1 and 6 now have four communication links.

\subsection{Mathematical Model of the Proposed Control Method}

The proposed control methodology presented in this subsection consists of primary and secondary control levels with a hierarchical control structure to ensure an accurate reactive power sharing control among DG units under mismatched feeder line impedance conditions. The primary level consists of droop control, adaptive virtual impedance control, and dual voltage and current loops. In the secondary level, leaderless and leader-followers consensus control algorithms are designed to update the primary level. An analytical study of reactive power sharing is considered since the proposed control aims to provide accurate reactive power sharing among DG units in an islanded microgrid. The stability of the reactive power sharing based on consensus control is explored and proven to be asymptotically stable.

Figure 6, below, illustrates the overall structure of the hierarchical control. The measured output voltage and current are converted into $d q 0$ frame and then used to calculate the active and reactive power. The power quantities are used to regulate frequency and voltage and then adaptive virtual impedance control adjust the voltage regulation in order to adjust the reference voltage for the inverter. The $d q 0$ quantity is converted back to the $a b c$ frame based on the reference frequency and voltages and then go through PI voltage and current loops to Sinusoidal Pulse Width Modulation (SPWM) in order to feed the inverter, as shown in Figure 6. Each DG unit and its primary control are connected to other DG units in a ring feeder distribution system, as shown in Figure 1. In Figure 6, the consensus control is considered to be a secondary control that communicates between DG units in order to exchange the information. In this figure, blue arrow indicates physical lines, black arrow indicates the primary control connections, and dark red arrow indicates communication links. In this paper, consensus control is implemented under three different communication topologies, the ring, complete, and triangle mesh. In this system, the feeder lines connect the DG units to supply the power demand. In the following, each part of this proposed control scheme explains in detail.

\subsubsection{Primary Control}

The primary level of hierarchical control consists of (1) droop control, (2) virtual impedance/adaptive virtual impedance control, and (3) outer voltage and inner current dual loop control. First, a droop control is applied to enable the active and reactive power sharing without communication links [1-9]. Each DG unit has a local droop control to regulate both active and reactive power to the reference values. Second, virtual impedance control balances the mismatched output impedance of the neighboring DG units to achieve better power sharing performance [1-4,8,12,13,15,25]. Third, dual loop voltage and current control is employed in order to compensate any deviation of the generated voltage and frequency to enhance the overall power quality [3-6]. 


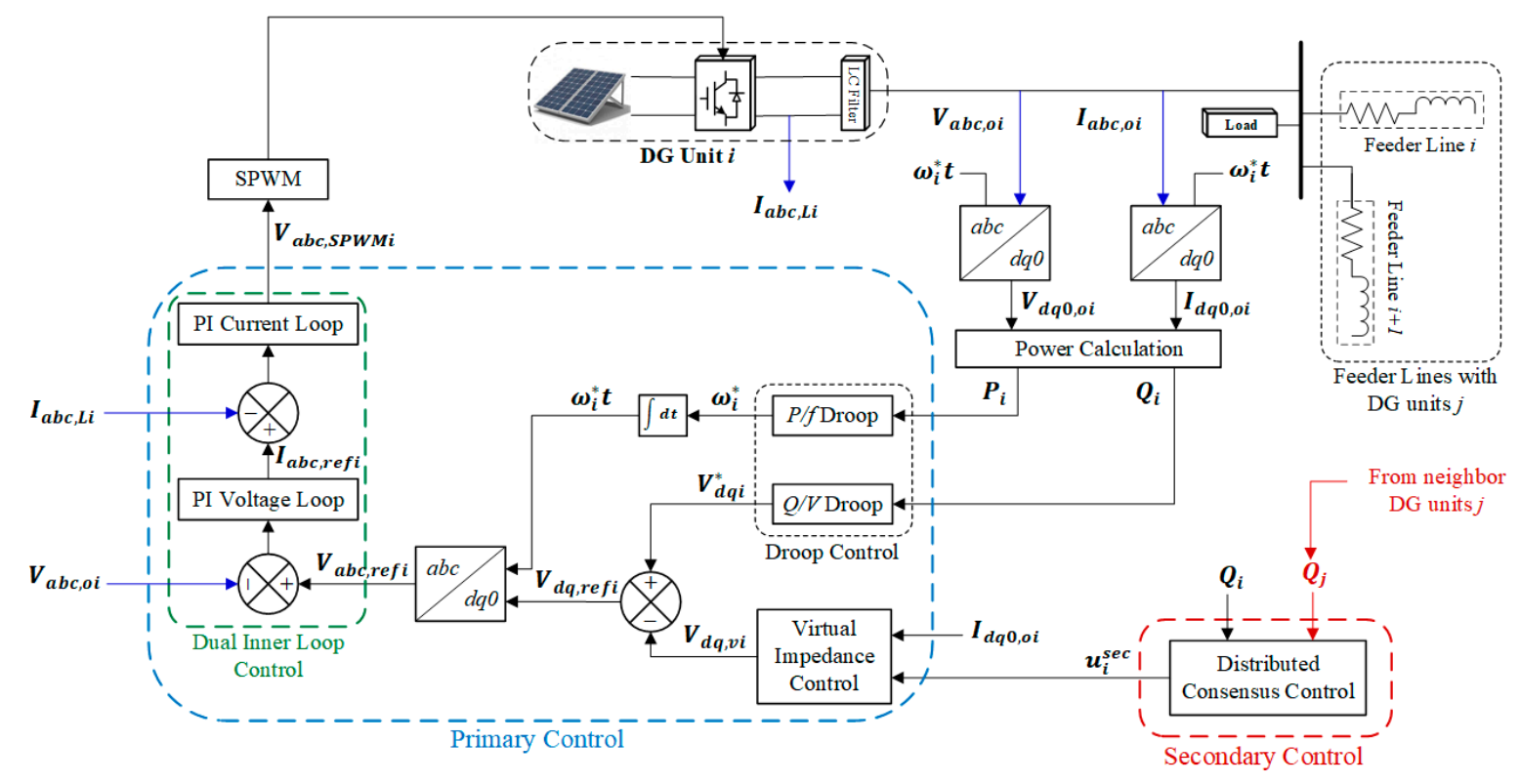

Figure 6. The scheme of the proposed control.

\section{Droop Control}

This classical approach to control in large power systems, where the analysis assumes a balanced three phased system with long transmission line models, typically assumes that the line impedances satisfy the condition $X_{i j} \gg R_{i j}$.

In this proposed control, the droop control is designed based on a mainly inductive feeder lines impedance, $X_{i j} \gg R_{i j}$. The phase angle difference $\left(\delta_{i}\right)$ between the inverter voltage $V_{a b c, o i}$ and the voltage of the load is assumed to be small. Thus, it can be assumed that $\cos \delta_{i}=1$ and $\sin \delta_{i}=\delta_{i}$. The active $\left(P_{i}\right)$ and reactive $\left(Q_{i}\right)$ power injections can then be written as

$$
P_{i}(t)=\sum_{j \in N_{i}}\left[\frac{\left(V_{a b c, o i}(t) V_{a b c, o j}(t) \delta_{i} \sin \left(\theta_{i}-\theta_{j}\right)\right)}{X_{i j}}\right]
$$

and

$$
Q_{i}(t)=\sum_{j \in N_{i}}\left[\frac{\left(V_{a b c, o i}(t) V_{a b c, o j}(t) \cos \left(\theta_{i}-\theta_{j}\right)-V_{a b c, o j}^{2}(t)\right)}{X_{i j}}\right]
$$

From Equations (1) and (2), it can be seen that the active power is related to power angle $\left(\delta_{i}\right)$, which is related to the output voltage frequency and the reactive power is related to the difference in the magnitude of output voltage of the DG units $[3,4,26]$. Thus, it is possible to use active power to adjust the frequency and reactive power to adjust the magnitude of the output voltage. These two relationships, which are commonly known as droop control and the angular frequency and voltage magnitude of the droop control, are expressed as the familiar droop control laws [1-9,26],

$$
\left\{\begin{array}{l}
\omega_{i}^{*}(t)=\omega_{n i}-k_{p i} P_{i}(t) \\
V_{a b c i}^{*}(t)=V_{n i}-k_{q i} Q_{i}(t)
\end{array}\right.
$$

where $\omega_{n i}$ and $V_{n i}$ are the nominal values of angular frequency and voltage amplitude respectively. $k_{p i}$ and $k_{q i}$ are the droop control coefficients of active power and reactive power, respectively. The droop 
control here is designed based on rotating reference frame quantities $(d q 0)$. Thus, Equation (3) can be expressed in the $(d q 0)$ frame, as

$$
\left\{\begin{array}{l}
\omega_{i}^{*}(t)=\omega_{n i}-k_{p i} P_{i}(t) \\
V_{d i}^{*}(t)=V_{n i}-k_{q i} Q_{i}(t) \\
V_{q i}^{*}(t)=0 .
\end{array}\right.
$$

These active power/frequency $\left(P_{i} / \omega_{i}\right)$ and reactive power/voltage $\left(Q_{i} / V_{i}\right)$ relations are appropriate when the output inverter impedance and the feeder line impedance are purely inductive. However, this assumption that is justified for a high-voltage system is impractical and difficult to ensure in low-voltage systems, such as islanded microgrids [1-4]. In the latter case, mismatched feeder line conditions often exist and the reactive power/voltage $\left(Q_{i} / V_{i}\right)$ relation exhibits poor reactive power sharing $[1-16,27]$.

\section{Virtual Impedance Control}

The virtual impedance loop ensures a consistent output impedance for all inverters in the islanded microgrid $[1-4,8,12,13,15,25]$. The feedback loop is employed here to improve the current regulation and change the effective output impedance of the inverters to arbitrary impedance, reducing the impedance mismatch.

A virtual impedance $\left(Z_{v}\right)$ is added between the output of the DG unit and the outer voltage loop in each unit providing an additional control input into the outer voltage loop to match the output impedance in all DG units. This virtual impedance effectively increases the output impedance of each DG unit to match with other impedances in all DG units. The mismatched feeder line effects are eliminated and the reactive power/voltage $\left(Q_{i} / V_{i}\right)$ relation in the droop control can improve reactive power sharing $[8,12]$. The proposed control is designed based on rotating reference frame quantities $(d q 0)$, and the voltage deviation of the virtual impedance control $\left(V_{d q, v}\right)$ can be expressed as

$$
V_{d q, v i}(t)=\left[\begin{array}{c}
V_{d, v i}(t) \\
V_{q, v i}(t)
\end{array}\right]=\underbrace{\left[\begin{array}{cc}
R_{v i} & X_{v i} \\
-X_{v i} & R_{v i}
\end{array}\right]}_{Z_{v}}\left[\begin{array}{c}
i_{o d i}(t) \\
i_{o q i}(t)
\end{array}\right]
$$

where $i_{o d}$ and $i_{o q}$ are the output current of the DG unit in $(d q 0)$ frame quantities, and $R_{v}$ and $X_{v}$ are the resistance and reactance values of the virtual impedance. Here, the voltage deviation in the virtual impedance control is employed in order to adjust the magnitude of the output voltage by modifying the voltage magnitude of the droop control $[8,12,13,15]$. The reference voltage $\left(V_{d q, r e f}\right)$ implemented in the outer voltage loop can be expressed as

$$
V_{d q, r e f i}(t)=V_{d q, i}^{*}(t)-V_{d q, v i}(t)
$$

Subsisting Equations (4) and (5) into Equation (6), the reference voltage $\left(V_{d q, r e f}\right)$ can be expressed as

$$
V_{d q, r e f i}(t)=\left[\begin{array}{l}
V_{d, r e f i}(t) \\
V_{q, r e f i}(t)
\end{array}\right]=\left[\begin{array}{c}
V_{n i}-k_{q i} Q_{i}(t) \\
0
\end{array}\right]-\underbrace{\left[\begin{array}{cc}
R_{v i} & X_{v i} \\
-X_{v i} & R_{v i}
\end{array}\right]}_{Z_{v i}}\left[\begin{array}{c}
i_{o d i}(t) \\
i_{o q i}(t)
\end{array}\right] .
$$

\section{Outer Voltage and Inner Current Dual Loop Control}

Outer voltage and inner current loops both have high bandwidth and fast response to any disturbances in the DG unit $[3,4,26]$. In this proposed control method, both control loops are designed 
in the synchronous reference frame $(a b c)$ and used Proportional-Integral (PI) controllers. The output current reference of outer loop $\left(I_{a b c, r e f}\right)$ is expressed as

$$
I_{a b c, r e f i}(t)=k_{p v}\left(V_{a b c, r e f i}(t)-V_{a b c, o i}(t)\right)+k_{i v} \int\left(V_{a b c, r e f i}(t)-V_{a b c, o i}(t)\right) d t,
$$

where $k_{p v}$ and $k_{i v}$ are the outer voltage loop parameters of the PI control. In the inner current loop, a SPWM modulated voltage signal $\left(V_{a b c, S P W M}\right)$ is obtained by comparing the inductor current $\left(I_{a b c, L i}\right)$ and the reference current $\left(I_{a b c, r e f}\right)$. The $V_{a b c, S P W M}$ is determined as

$$
V_{a b c, S P W M}(t)=k_{p i}\left(I_{a b c, r e f}(t)-I_{a b c, L i}(t)\right)+k_{i i} \int\left(I_{a b c, r e f}(t)-I_{a b c, L i}(t)\right) d t,
$$

where $k_{p i}$, and $k_{i i}$ are the inner current loop parameters of the PI control.

\subsubsection{Secondary Control}

Based on previous studies, the virtual impedance control method requires accurate knowledge of the DG and feeder line parameters to accurately balance the mismatched output impedance among DG units, which is not easy to guarantee in practical applications $[1-4,8,12,13,15,25]$. A secondary control is employed to restore the frequency and voltage by providing reference values to the primary control level to eliminate the inherent frequency and voltage deviations to overcome this challenge [3]. A consensus control method is designed in this proposed control to regulate voltage and ensure accurate reactive power to overcome the reactive power/voltage $\left(Q_{i} / V_{i}\right)$ droop relation limitation associated with the mismatched feeder line condition.

\section{Reactive Power Sharing}

In order to ensure the reactive power sharing among DG units under mismatched feeder line conditions, a proportional reactive power sharing among DG units should be achieved, such that $[17,25,34]$

$$
k_{q 1} Q_{1}=k_{q 2} Q_{2}=\ldots=k_{q N-1} Q_{N-1}=k_{q N} Q_{N},
$$

where $n=1,2, \ldots, N$. The secondary control should achieve this proportional reactive power sharing condition to ensure accurate reactive power sharing. With the reactive power/voltage $\left(Q_{i} / V_{i}\right)$ droop relation substituted in Equation (2), the proportional reactive power sharing can be expressed as

$$
k_{q i} Q_{i}(t)=\sum_{j \in N_{i}}\left[\frac{V_{a b c, o j}(t)\left(V_{n i} \cos \left(\theta_{i}-\theta_{j}\right)-V_{a b c, o j}(t)\right)}{\frac{X_{i j}}{k_{q i}}+V_{a b c, o j}(t) \cos \left(\theta_{i}-\theta_{j}\right)}\right] .
$$

From Equation (11), the following equivalent equation can be obtained,

$$
\frac{X_{1 j}}{k_{q 1}}=\frac{X_{2 j}}{k_{q 2}}=\ldots=\frac{X_{N-1 j}}{k_{q N-1}}=\frac{X_{N j}}{k_{q N}} .
$$

From (12), we note that $k_{q i}$ should be selected based on its propositional relationship with its corresponding line reactance. In the mismatched feeder line case, droop control cannot control the reactive power, since $k_{q i}$ is fixed in droop control, while in this case, $k_{q i}$ needs to be updated or adjusted based on how the mismatched feeder line influences the system performance [25,28-30,34]. Designing a secondary control to ensure accurate reactive power sharing expressed in Equation (10) requires an adaptive virtual impedance control method based on consensus control, such a control is proposed here. 
Adaptive Virtual Impedance Control

To achieve consistent or equivalent output impedance for all inverters in the islanded microgrid, an adaptive control term is added to provide an online update into the virtual impedance. The virtual impedance control in Equation (5) can be rewritten to include the adaptive virtual impedance, as

$$
V_{d q, v i}(t)=\left[\begin{array}{c}
V_{d, v i}(t) \\
V_{q, v i}(t)
\end{array}\right]=\underbrace{\left[\begin{array}{cc}
R_{v i} & X_{v i} \\
-X_{v i} & R_{v i}
\end{array}\right]}_{Z_{v i}}\left[\begin{array}{c}
i_{o d i}(t) \\
i_{o q i}(t)
\end{array}\right] \times \underbrace{\left[\begin{array}{c}
u_{i}^{\text {sec }}(t) \\
u_{i}^{\text {sec }}(t)
\end{array}\right]}_{\text {adaptive term }},
$$

where $u_{i}^{\text {sec }}$ is an adaptive term that is updated based on consensus control. Figure 7 illustrates the process of updating this adaptive virtual impedance.

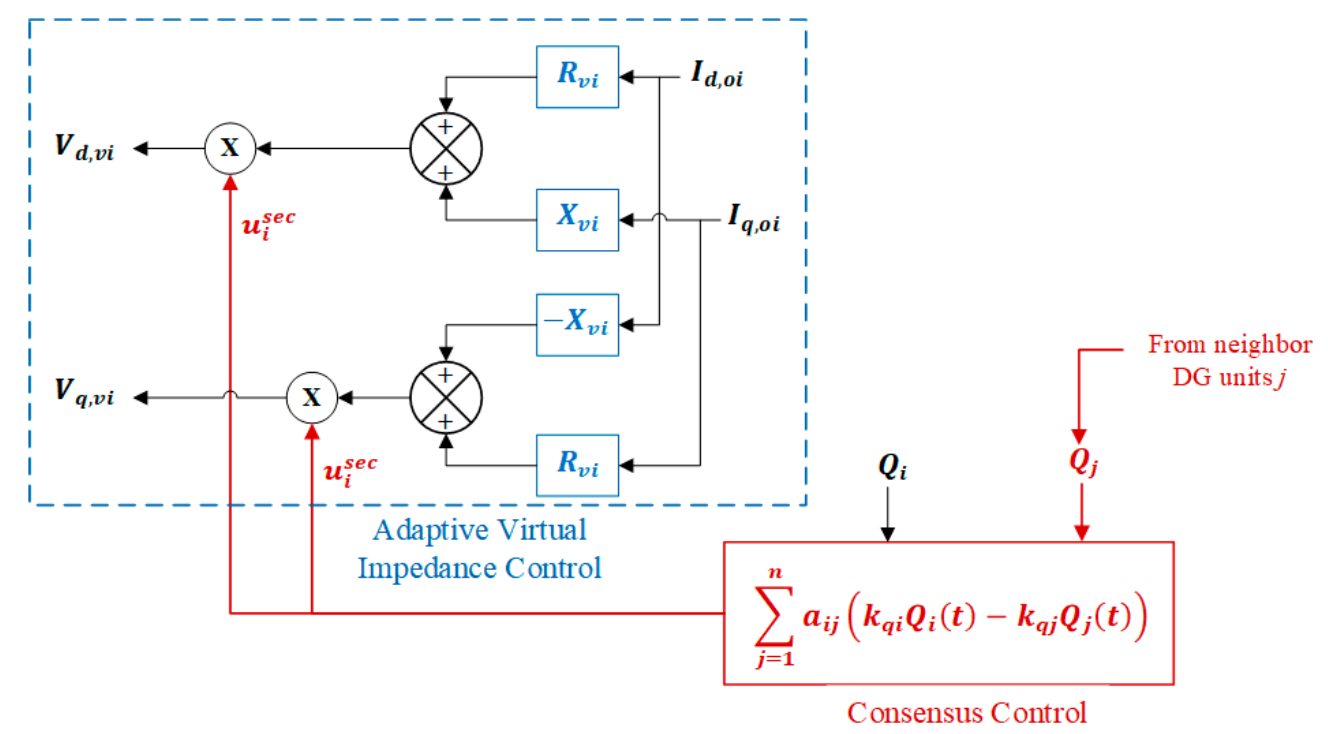

Figure 7. A scheme of adaptive virtual impedance control.

The reference voltage $\left(V_{d q, r e f}\right)$ in Equation (7) can be rewritten as

$$
\left[\begin{array}{c}
V_{d, \text { refi }}(t) \\
V_{q, \text { refi }}(t)
\end{array}\right]=\left[\begin{array}{c}
V_{n i}-k_{q i} Q_{i}(t) \\
0
\end{array}\right]-\underbrace{\left[\begin{array}{cc}
R_{v i} & X_{v i} \\
-X_{v i} & R_{v i}
\end{array}\right]}_{Z_{v i}}\left[\begin{array}{c}
i_{o d i}(t) \\
i_{\text {oqi }}(t)
\end{array}\right] \times \underbrace{\left[\begin{array}{c}
u_{i}^{\text {sec }}(t) \\
u_{i}^{\sec }(t)
\end{array}\right]}_{\text {adaptive term }} .
$$

This adaptive virtual impedance ensures an inductive DG equivalent feeder reactance by adding $Z_{v i}$, and the reactive power sharing can be guaranteed without knowledge of the feeder line impedances.

\section{Consensus Control}

Using a consensus control protocol as a secondary control level enables each DG unit to update its reference values based on its neighbors' values [22,23,29,30,35-37]. Each DG unit converges to a certain consensus point. In this proposed control, the distributed consensus control is designed in order to solve a regulator synchronization problem for reactive power sharing. The control is considered as a first-order linear multi-agent system (MAS), which can be expressed as

$$
\left\{\begin{array}{l}
\dot{x}_{i}(t)=\mathcal{A} x_{i}(t) \\
\mathcal{A} x_{i}(t)=u_{i}(t),
\end{array}\right.
$$


where $i=\{1,2, \ldots, n\}$, and $x_{i} \in R^{n}$ and $u_{i} \in \boldsymbol{R}^{n}$ are the state and input control of DG unit $i$ and $\mathcal{A}$ donates the adjacency matrix of the communication graph, where $\mathcal{A}=\left[a_{i j}\right] \in \boldsymbol{R}^{n x n}$.

Theorems and Lemmas

The global dynamics of system and input control in (15) can be defined as

$$
\left\{\begin{array}{l}
\dot{X}(t)=\mathcal{A} X(t) \\
\mathcal{A} X(t)=U(t)
\end{array}\right.
$$

where $X$ and $U$ are the global state and input control of the MAS system, respectively. Here, $X \in R^{n N}$ and $\boldsymbol{U} \in \boldsymbol{R}^{n N}$, where $\boldsymbol{n}$ donates the number of states in each DG unit and $N$ donates the number of DG units in the entire system. In addition to the adjacency matrix $(\mathcal{A})$, the communication graph can be presented by a diagonal matrix of in degrees $\mathcal{D}=\operatorname{diag}\left\{d_{i}\right\} \in R^{n x n}$ and Laplacian matrix $\mathcal{L}=\left[l_{i j}\right] \in R^{n x n}$. The relation among these communication graph matrices can be expressed and Laplacian matrix $\mathcal{L}$ can be defined as

$$
\mathcal{L}=\mathcal{D}-\mathcal{A}=\left\{\begin{array}{l}
l_{i j}=-a_{i j}, \quad \text { when } i \neq j \text { and } i \text { and } j \text { are connected } \\
l_{i j}=0, \text { when } i \neq j \text { and } i \text { and } j \text { are not connected } \\
l_{i j}=\sum_{j=1}^{n} a_{i j} .
\end{array}\right.
$$

All row-sums of $\mathcal{L}$ are zero $\left(\sum_{j} l_{i j}=0\right)$, according to the definition of the Laplacian matrix of a communication graph $\mathcal{L}$ in (17). This guarantees that $\mathcal{L}$ always has one and only one zero-eigenvalue $\left(\lambda_{1}=\mathbf{0}\right)$ and the rest of the eigenvalues are positive. Therefore, the Laplacian matrix of a communication graph $\mathcal{L}$ is a positive semidefinite matrix.

Theorem 1. Consensus or agreement can be converged if the following condition is achieved: (1) Laplacian matrix of a communication graph $(\mathcal{L})$ has one and only one zero-eigenvalue while the rest of the eigenvalues are positive and (2) if and only if a spanning tree is available in the communication graph [34].

Theorem 2. Every connected graph has at least one spanning tree. Let the communication graph is a connected graph, if it has no cycle, then it is its spanning tree. In case it has cycles, then deleting one edge from the cycles does not affect the existence of spanning tree and the communication graph remains connected and cycle free containing all the agents of the communication graph [32].

Theorem 3. The states of all DG units will converge to the leader state if and only if a spanning tree with root node $i \in B$ is available in the communication graph [32].

Using Theorem 1, all of the states can converge to the consensus if $\mathcal{L}$ is positive semidefinite (17). In addition, a spanning tree should be available in the communication topology. Using Theorem 2, the communication topology has at least one spanning tree as long as $\mathcal{G}$ remains connected.

Some technical lemmas are necessary to design a stable consensus control.

Lemma 1. The Laplacian matrix for the undirected communication graph satisfies the following identity [38]:

$$
2 X^{T} \mathcal{L} X=\sum_{i=1}^{n} \sum_{j=1}^{n} a_{i j}\left(x_{i}-x_{j}\right)^{2} .
$$

Proof of Lemma 1. Expand $X^{T} \mathcal{L} X$ is given by

$$
X^{T} \mathcal{L} X=\sum_{i=1}^{n} \sum_{j=1}^{n} l_{i j} x_{i} x_{j}
$$


In case $i=j$, then $x_{i}=x_{j}$ otherwise $x_{i} \neq x_{j}$. so,

$$
X^{T} \mathcal{L} X=\sum_{i=1}^{n}\left(l_{i i} x_{i}^{2}\right)+\left(\sum_{j=1, i \neq j}^{n} l_{i j} x_{i} x_{j}\right) .
$$

According to (17), Equation (20) can be rewritten as

$$
X^{T} \mathcal{L} X=\sum_{i=1}^{n}\left(x_{i}^{2} \sum_{j=1}^{n} a_{i j}\right)-\left(\sum_{j=1,}^{n} a_{i j} x_{i} x_{j}\right),
$$

thus,

$$
X^{T} \mathcal{L} X=\sum_{i=1}^{n} \sum_{j=1,}^{n} a_{i j}\left(x_{i}^{2}-x_{i} x_{j}\right)
$$

Assume that the communication topology here is undirected, so $a_{i j}=a_{j i} . X^{T} \mathcal{L} X$ can be written as:

$$
X^{T} \mathcal{L} X=\sum_{i=1}^{n} \sum_{j=1}^{n} a_{i j}\left(x_{i}^{2}-x_{i} x_{j}\right)=\sum_{i=1}^{n} \sum_{j=1}^{n} a_{j i}\left(x_{j}^{2}-x_{j} x_{i}\right) .
$$

To complete the proof, $2 X^{T} \mathcal{L} X$ is calculated as

$$
2 X^{T} \mathcal{L} X=\sum_{i=1}^{n} \sum_{j=1,}^{n} a_{i j}\left(x_{i}^{2}-x_{i} x_{j}\right)+\sum_{i=1}^{n} \sum_{j=1,}^{n} a_{j i}\left(x_{j}^{2}-x_{j} x_{i}\right) .
$$

Simplify (24), then

$$
2 X^{T} \mathcal{L} X=\sum_{i=1}^{n} \sum_{j=1}^{n} a_{i j}\left(x_{i}^{2}-2 x_{i} x_{j}+x_{j}^{2}\right)=\sum_{i=1}^{n} \sum_{j=1}^{n} a_{i j}\left(x_{i}-x_{j}\right)^{2},
$$

and proof of Lemma 1 is completed.

Lemma 2. If an undirected communication graph is a connected graph, then the rank of the Laplacian matrix $\operatorname{rank}(\operatorname{rank}(\mathcal{L})=N-\mathbf{1})[39]$.

Lemma 3. If $\mathcal{L}=\mathcal{L}^{T} \in R^{n x n}$, then, the null space of the Laplacian matrix of the communication graph is $\operatorname{null}(\mathcal{L})=\left\{x \in \boldsymbol{R}^{n} \mid x^{T} \mathcal{L} x=0\right\}$ if and only if $\mathcal{L}$ is positive semidefinite $(\mathcal{L} \geq \mathbf{0})$ or negative semidefinite $(\mathcal{L} \leq \mathbf{0})[38]$.

Lemma 4. For a connected undirected communication graph, $2 X^{T} \mathcal{L} X=0$, if and only if the argument or consensus of MAS system is achieved, which occurs when $x_{i}=x_{j}$ for $\forall i, j=\{1,2, \ldots, n\}$ [38].

Proof of Lemma 4. if $2 X^{T} \mathcal{L} X=0$, then

$$
\sum_{i=1}^{n} \sum_{j=1,}^{n} a_{i j}\left(x_{i}-x_{j}\right)^{2}=0,
$$

which implies $x_{i}=x_{j}$ for $\forall i, j=\{1,2, \ldots, n\}$. Alternatively, because the communication graph is undirected, $\mathcal{L}$ is symmetric and positive semidefinite $(\mathcal{L} \geq \mathbf{0})$. Using Lemma 3, the null space of the Laplacian matrix of the communication graph is $\operatorname{null}(\mathcal{L})=\left\{x \in R^{n} \mid x^{T} \mathcal{L} x=0\right\}$. Here, $\mathcal{L}$ has one and only one zero-eigenvalue and the other eigenvalues are positive. Thus, $\mathcal{L}$ has an eigenvector of $1_{N}=[1,1, \ldots, N] \in \boldsymbol{R}^{N}$. Using Lemma 2 , since the communication graph here is designed to be undirected, the $(\operatorname{rank}(\mathcal{L})=N-\mathbf{1})$. Thus, the dimension of null space of $\mathcal{L}$ is $1,\left\{X \mid X=\alpha 1_{N}, \forall \alpha \in \boldsymbol{R}\right\}$, and the proof of Lemma 4 is complete.

Lemma 5. If the undirected communication graph is connected, then the consensus of the system in (16) is reached using the control law in (18) using the result in Lemma 1 [38]. 
Proof of Lemma 5. Consider the Lyapunov candidate, $\boldsymbol{V}=\sum_{i=1}^{N} x_{i}^{2}=X^{T} X$, then $\dot{V}=2 X^{T} X=-2 X^{T} \mathcal{L} X$. Using Lemma $1, \dot{V}$ depends on the Laplacian matrix $\mathcal{L}$ to ensure the stability of the system.

Reactive Power Sharing Based on Consensus Control

In order to represent the dynamics of the system using proportional reactive power sharing terminology, Equation (16) can be rewritten as

$$
\underbrace{k_{q N} \dot{Q}_{N}(t)}_{\dot{X}(t)}=\mathcal{A} \underbrace{k_{q N} Q_{N}(t)}_{X(t)}=\underbrace{u_{N}^{s e c}(t)}_{U(t)} .
$$

The reactive power errors among DG units should be eliminated to reach the consensus region or consensus point [34]. The consensus control algorithm for a $i^{\text {th }} \mathrm{DG}$ unit can be expressed, as

$$
u_{i}^{s e c}(t)=-C_{k Q i} \cdot e_{k Q i}
$$

where $C_{k Q i}$ donates coupling gain and $e_{k Q i}$ represents the local neighbor reactive power sharing error that can be expressed as

$$
e_{k Q i}(t)=\sum_{j=1}^{n} a_{i j}\left(k_{q i} Q_{i}(t)-k_{q j} Q_{j}(t)\right),
$$

where $k_{q i} Q_{i}(t)$ and $k_{q j} Q_{j}(t)$ are the proportional reactive power outputs of DG unit $i$ and its neighbors, respectively. The entire system can be written in matrix form as

$$
\left[\begin{array}{c}
k_{q 1} \dot{Q}_{1} \\
k_{q 2} \dot{Q}_{2} \\
\vdots \\
k_{q N} \dot{Q}_{N}
\end{array}\right]=\left[\begin{array}{c}
u_{1}^{s e c}(t) \\
u_{2}^{s e c}(t) \\
\vdots \\
u_{N}^{s e c}(t)
\end{array}\right]=\left[\begin{array}{c}
-C_{k Q 1} \\
-C_{k Q 2} \\
\vdots \\
-C_{k Q N}
\end{array}\right] \times \underbrace{\sum_{j=1}^{n}\left[\begin{array}{c}
a_{1 j} \\
a_{2 j} \\
\vdots \\
a_{N j}
\end{array}\right] \times\left[\begin{array}{c}
\left(k_{q 1} Q_{1}(t)-k_{q j} Q_{j}(t)\right) \\
\left(k_{q 2} Q_{2}(t)-k_{q j} Q_{j}(t)\right) \\
\vdots \\
\left(k_{q N} Q_{N}(t)-k_{q j} Q_{j}(t)\right)
\end{array}\right]}_{e_{k Q N}(t)}
$$

where

$$
\left\{\begin{array}{l}
k_{q N} \dot{Q}_{N}=u_{d N}^{s e c}(t) \\
u_{N}^{s c}(t)=-C_{k Q N} \times e_{k Q N}(t), \\
e_{k Q N}=\mathcal{L} \times k_{q N} Q_{N}(t) .
\end{array},\right.
$$

and, the entire system can be expressed as

$$
k_{q N} \dot{Q}_{N}=-C_{k Q N} \times \mathcal{L} \times k_{q N} Q_{N}(t) .
$$

Equation (29) represents the leaderless consensus control algorithm. In the case of leader-followers consensus control, a DG unit is selected to be a leader and other DG units as its followers $[34,36,37]$. Thus, Equation (29) can be rewritten to describe this leader-follower structure, as

$$
e_{k Q i}(t)=\sum_{j=1}^{n} a_{i j}\left(k_{q i} Q_{i}(t)-k_{q j} Q_{j}(t)\right)-b_{i}\left(k_{q i} Q_{i}(t)-k_{q \text { Leader }} Q_{\text {Leader }}(t)\right)
$$

where

$$
\left\{\begin{array}{l}
b_{i}=1, \quad \text { if }(i) \text { is the leader } \\
b_{i}=0, \quad \text { if }(i) \text { is not the leader, }
\end{array}\right.
$$

and $k_{\text {qLeader }} Q_{\text {Leader }}$ denotes to the proportional reactive power sharing of the DG unit selected to lead the system. 
Stability Analysis of Consensus Control

The stability analysis of the reactive power consensus control is now developed. From the literature $[17,28]$, the linear consensus control protocol can be expressed, as

$$
\left\{\begin{array}{l}
\underbrace{k_{q N} \dot{Q}_{N}}_{\dot{X}_{e}}=u_{d N}^{s e c}(t) \\
\underbrace{u_{N}^{s e c}(t)}_{U}=-C_{k Q N} \times \mathcal{L} \times \underbrace{k_{q N} Q_{N}(t)}_{X_{e}} .
\end{array}\right.
$$

Using Lemma 1 and Lemma 5, the Lyapunov function candidate is selected as $V_{e}=X_{e}^{T} X_{e}$, and the derivative of this candidate is $\dot{V}_{e}=2 X_{e}^{T} \dot{X}_{e}=-2 C_{k Q N} X_{e}^{T} \mathcal{L} X_{e}$ [40]. It is obvious that the stability of the system depends on $\mathcal{L}$ [38]. $\mathcal{L}$ is a positive semidefinite matrix, according to the definition of the Laplacian matrix of a communication graph $\mathcal{L}$ in (17). Thus, the consensus control in (35) is Lyapunov stable [40]. This stability analysis is based on the eigenvalues of the $\mathcal{L}$. In order to improve this finding, a quadratic candidate Lyapunov function is selected as

$$
\boldsymbol{V}=X_{e}^{T} P X_{e}
$$

where $\boldsymbol{P} \in \boldsymbol{R}^{n x n}, \boldsymbol{P}>\mathbf{0}$ is positive definite and $\boldsymbol{P}>\boldsymbol{P}^{T}$. Subsequently, the derivative of this Lyapunov candidate is

$$
\dot{V}_{e}=2 X_{e}^{T} P \dot{X}_{e}=-2 C_{k Q N} X_{e}^{T}(P \mathcal{L}) X_{e} .
$$

The derivative in (37) can be written as

$$
\dot{\boldsymbol{V}}_{\boldsymbol{e}}=-2 C_{k Q N} X_{e}^{T}\left(\mathcal{L}^{T} \boldsymbol{P}+\boldsymbol{P} \mathcal{L}\right) X_{e} .
$$

From [34], the $\mathcal{L}^{T} \boldsymbol{P}+\boldsymbol{P} \mathcal{L}$ term is positive definite, which means that $\dot{V}_{e}<\boldsymbol{0}$ is negative definite. Using Lyapunov theory, candidate $\boldsymbol{P}$ in (36) shows that the proposed control is asymptotically stable [40]. However, the $\mathcal{L}^{T} \boldsymbol{P}+\boldsymbol{P} \mathcal{L}$ term depends on the selection of $\boldsymbol{P}$, since $\mathcal{L}$ is guaranteed to be positive semidefinite by definition (17). In a system such as an islanded microgrid, the expansion of the system is expected, which makes selecting values of the $\boldsymbol{P}$ matrix difficult and impractical. Therefore, alternative methods for guaranteeing asymptotically stable are now proposed.

From the analysis above, it is evident that the Laplacian matrix of the communication graph plays a key role in guaranteeing Lyapunov stability. The connectivity of the communication graph must be taken into consideration to ensure stability [38]. Here, the adjacency $(\mathcal{A})$ and Laplacian $(\mathcal{L})$ matrices are irreducible, and the quadratic Lyapunov function is difficult to select in order to ensure asymptotic stability [38]. Therefore, an alternative method is proposed to guarantee an asymptotically stable system. Here, the input control in Equation (35) is modified and expressed as

$$
\underbrace{u_{N}^{s e c}(t)}_{U}=-C_{k Q N} \times(\mathcal{L}+\mathcal{B}) \times \underbrace{k_{q N} Q_{N}(t)}_{X_{e}},
$$

where $\mathcal{B} \in R^{n x n}$ is a diagonal matrix with nonnegative diagonal entities and at least one of them is positive [38]. This matrix guarantees that the $(\mathcal{L}+\mathcal{B})$ matrix is nonsingular. In other words, $(\mathcal{L}+\mathcal{B})$ matrix is positive definite. Therefore, the Lyapunov function candidate that is selected above as $V_{e}=X_{e}^{T} X_{e}$ and its derivative $\dot{V}_{\boldsymbol{e}}=-2 C_{k Q N} X_{e}^{T}(\mathcal{L}+\mathcal{B}) X_{e}$ is asymptotically stable, since $(\mathcal{L}+\mathcal{B})$ matrix is guaranteed to be positive definite $[38,40]$. In addition, selecting the quadratic Lyapunov 
function in Equation (36) and using linear quadratic Lyapunov Equation in (38), the derivative of linear quadratic Lyapunov candidate can be written, as

$$
\dot{\boldsymbol{V}}_{\boldsymbol{e}}=-2 C_{k Q N} X_{e}^{T}\left((\mathcal{L}+\mathcal{B})^{T} \boldsymbol{P}+\boldsymbol{P}(\mathcal{L}+\mathcal{B})\right) X_{e}
$$

This alternative method reduces the proposed control's sensitivity to the $\boldsymbol{P}$ matrix selection and ensures asymptotic stability.

\section{Overall Control}

The consensus control updates the local adaptive virtual impedance controls in the primary level of each DG unit to eliminate the reactive power errors among the DG units. Thus, each DG adjusts its virtual impedance based on its neighbors until all DG units reach the consensus region or point. Substituting Equations (28), (29), and (39) in the Equation in (13), the overall control can be expressed, as

$$
\left[\begin{array}{c}
V_{d, v i}(t) \\
V_{q, v i}(t)
\end{array}\right]=\underbrace{\left[\begin{array}{cc}
R_{v i} & X_{v i} \\
-X_{v i} & R_{v i}
\end{array}\right]}_{Z_{v i}}\left[\begin{array}{c}
i_{o d i}(t) \\
i_{o q i}(t)
\end{array}\right] \times \underbrace{\left[\begin{array}{l}
-C_{k Q N}(\mathcal{L}+\mathcal{B}) \times \sum_{j=1}^{n} a_{i j}\left(k_{q i} Q_{i}(t)-k_{q j} Q_{j}(t)\right) \\
-C_{k Q N}(\mathcal{L}+\mathcal{B}) \times \sum_{j=1}^{n} a_{i j}\left(k_{q i} Q_{i}(t)-k_{q j} Q_{j}(t)\right)
\end{array}\right]}_{\text {adaptive term }} .
$$

The reference of the adaptive virtual impedance $\left(V_{d q, v i}(t)\right)$ adjusts the voltage reference of the droop control $\left(V_{d q i}^{*}(t)\right)$. Substituting Equations (4), and (41) in Equation (14), the reference voltage that feeds into the outer voltage loop can be rewritten, as

$$
\left[\begin{array}{c}
V_{d, r e f i}(t) \\
V_{q, r e f i}(t)
\end{array}\right]=\left[\begin{array}{c}
V_{n i}-k_{q i} Q_{i}(t) \\
0
\end{array}\right]+\underbrace{\left[\begin{array}{cc}
R_{v i} & X_{v i} \\
-X_{v i} & R_{v i}
\end{array}\right]}_{Z_{v i}}\left[\begin{array}{c}
i_{o d i}(t) \\
i_{o q i}(t)
\end{array}\right] \underbrace{\left[\begin{array}{c}
C_{k Q N}(\mathcal{L}+\mathcal{B}) \sum_{j=1}^{n} a_{i j}\left(k_{q i} Q_{i}(t)-k_{q j} Q_{j}(t)\right) \\
C_{k Q N}(\mathcal{L}+\mathcal{B}) \sum_{j=1}^{n} a_{i j}\left(k_{q i} Q_{i}(t)-k_{q j} Q_{j}(t)\right)
\end{array}\right]}_{\text {adaptive term }}
$$

\section{Results and Discussion}

This section confirms that the proposed control methodology provides accurate active and reactive power sharing among DG units in an islanded microgrid. Three communication topologies are examined under leaderless and leader-followers consensus control algorithms. Ring and complete communication topologies are compared to the triangle mesh communication topology. Five cases are implemented in order to verify the effectiveness of the triangle mesh communication topology.

A configuration of six DG units with the same capacity of $10 \mathrm{kVA}$ operated in a ring feeder distribution system, as shown in Figure 1. Each unit consists of a DC source, such as a PV array, a three-phase inverter, an LC filter, and a load. The line length between DG units 1 and 2 and DG units 5 and 6 were considered to be $100 \mathrm{~m}$, the lines length between DG units 1 and 3 and DG units 4 and 6 were considered to be $150 \mathrm{~m}$, and the lines length between DG units 2 and 4 and DG units 3 and 5 were considered to be $200 \mathrm{~m}$, to consider feeder line mismatch condition. The line impedance of $1 \mathrm{~km}$ is $\left(\boldsymbol{R}_{f d}=0.642 \Omega\right.$ and $\left.X_{f d}=0.083 \Omega\right)$. Table 1 presents the line impedance parameters. In this model, the DG units supply five balanced loads; three of them are constant power loads and the other two are constant impedance loads. The parameters of DG unit components and these loads are detailed in Tables 2 and 3, respectively. Table 3 displays in detail load locations, time of operation, and capacity. A constant power load (load 1) and constant impedance load (load 5) are connected over the entire simulation time window, and the other three loads switched on or off differently in order to examine the performance of the proposed control under power change conditions. Another constant power load (load 3) is connected from the beginning for two seconds, and a constant impedance load (load 4) switches on at $1 \mathrm{~s}$. Additionally, a constant power load (load 4) is connected at $3 \mathrm{~s}$. In the secondary 
level, the leaderless and leader-followers consensus control algorithms are designed in this simulation. The simulation model and results are developed in MATLAB/Simulink.

Table 1. The feeder line parameters.

\begin{tabular}{cccc}
\hline Feeder Line between & Length & Resistance & Inductance \\
\hline DGs 1 \& 2 & $1 \mathrm{~km}$ & $0.642 \Omega$ & $0.22 \mathrm{mH}$ \\
DGs 1 \& 3 & $1.5 \mathrm{~km}$ & $0.963 \Omega$ & $0.33 \mathrm{mH}$ \\
DGs 2 \& 4 & $2 \mathrm{~km}$ & $1.284 \Omega$ & $0.44 \mathrm{mH}$ \\
DGs 3 \& 5 & $2 \mathrm{~km}$ & $1.284 \Omega$ & $0.44 \mathrm{mH}$ \\
DGs 4 \& 6 & $1.5 \mathrm{~km}$ & $0.963 \Omega$ & $0.33 \mathrm{mH}$ \\
DGs 5 \& 6 & $1 \mathrm{~km}$ & $0.642 \Omega$ & $0.22 \mathrm{mH}$ \\
\hline
\end{tabular}

Table 2. Parameters of DG unit components and primary control.

\begin{tabular}{cccccc}
\hline DG Component & Parameters & Values & DG Component & Parameters & Values \\
\hline \multirow{2}{*}{ LC Filter } & $L_{f}$ & $4 \mathrm{mH}$ & Virtual Impedance & $R_{v i}$ & $0.01 \Omega$ \\
& $C_{f}$ & $100 \mu \mathrm{F}$ & & $X_{v i}$ & $0.5 \mathrm{mH}$ \\
\hline \multirow{3}{*}{ Droop Control } & $\omega_{n i}$ & $2 \pi \cdot 60 \mathrm{rad} / \mathrm{sec}$ & Outer Voltage Loop & $k_{p v}$ & 1.8 \\
& $V_{n i}$ & $311 \mathrm{~V}\left(220 V_{r m s}\right)$ & & $k_{i v}$ & 10 \\
\cline { 2 - 6 } & $k_{p i}$ & $5 \times 10^{-5}$ & Inner Current Loop & $k_{p i}$ & 1.8 \\
\hline DC sources & $k_{q i}$ & $7 \times 10^{-4}$ & & & 10 \\
\hline
\end{tabular}

Table 3. Loads parameters.

\begin{tabular}{ccccc}
\hline $\begin{array}{c}\text { Constant Power } \\
\text { Load }\end{array}$ & Load Location & Operation Time & Active Power & Reactive Power \\
\hline Load 1 & DG 1 & All time & $5 \mathrm{~kW}$ & $3 \mathrm{kVar}$ \\
Load 3 & DG 4 & From 0 to 2 s & $5 \mathrm{~kW}$ & $5 \mathrm{kVar}$ \\
Load 4 & DG 5 & From 3 to 4 s & $4 \mathrm{~kW}$ & $5 \mathrm{kVar}$ \\
\hline Constant & Load Location & Operation Time & Resistance & Inductance \\
Impedance Load & & From 1 to 4 s & $10 \Omega$ & $27 \mathrm{mH}$ \\
Load 2 & DG 3 & All time & $15 \Omega$ & $40.5 \mathrm{mH}$ \\
\hline
\end{tabular}

\subsection{Case Study}

\subsubsection{Case 1: Primary Control Alone}

In this first case, the primary control operates locally without any communication links between the DG units. Thus, each local control works independently based on its local information only. Figures 8 and 9 present the simulation results of frequencies and active power sharing. According to IEEE Std 1547.4-2011 [41], a DG unit in islanded mode has to be able to regulate the frequency within an acceptance band. In Figure 8, all DG units' frequencies are regulated at $59.85 \mathrm{~Hz}$, which is within the acceptance band in the period of $0-1 \mathrm{~s}$. There is a slight drop/raise in the other time periods based on the active power increase/decrease, as can be seen in Figures 8 and 9. 


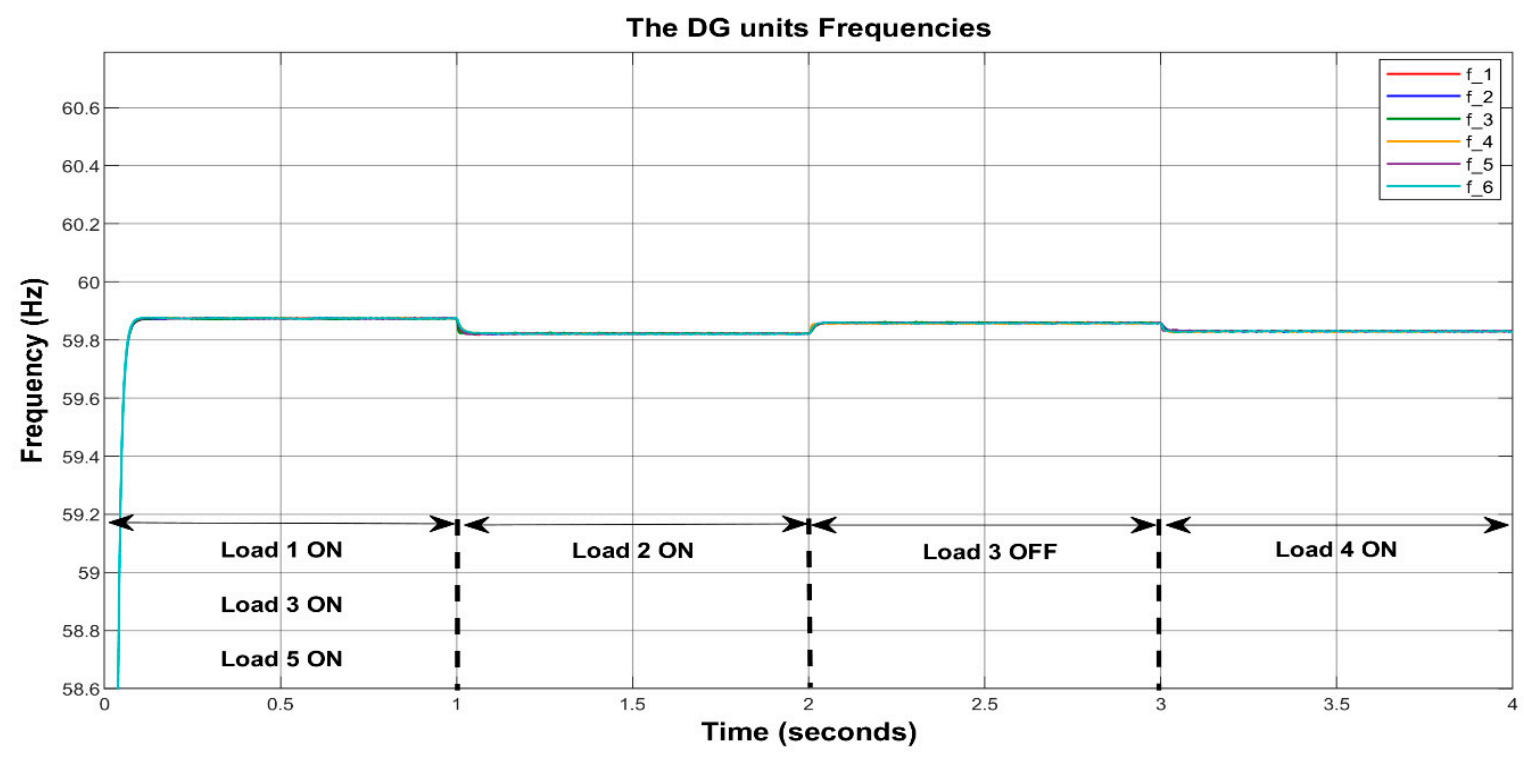

Figure 8. The DG units' frequencies for Case 1.

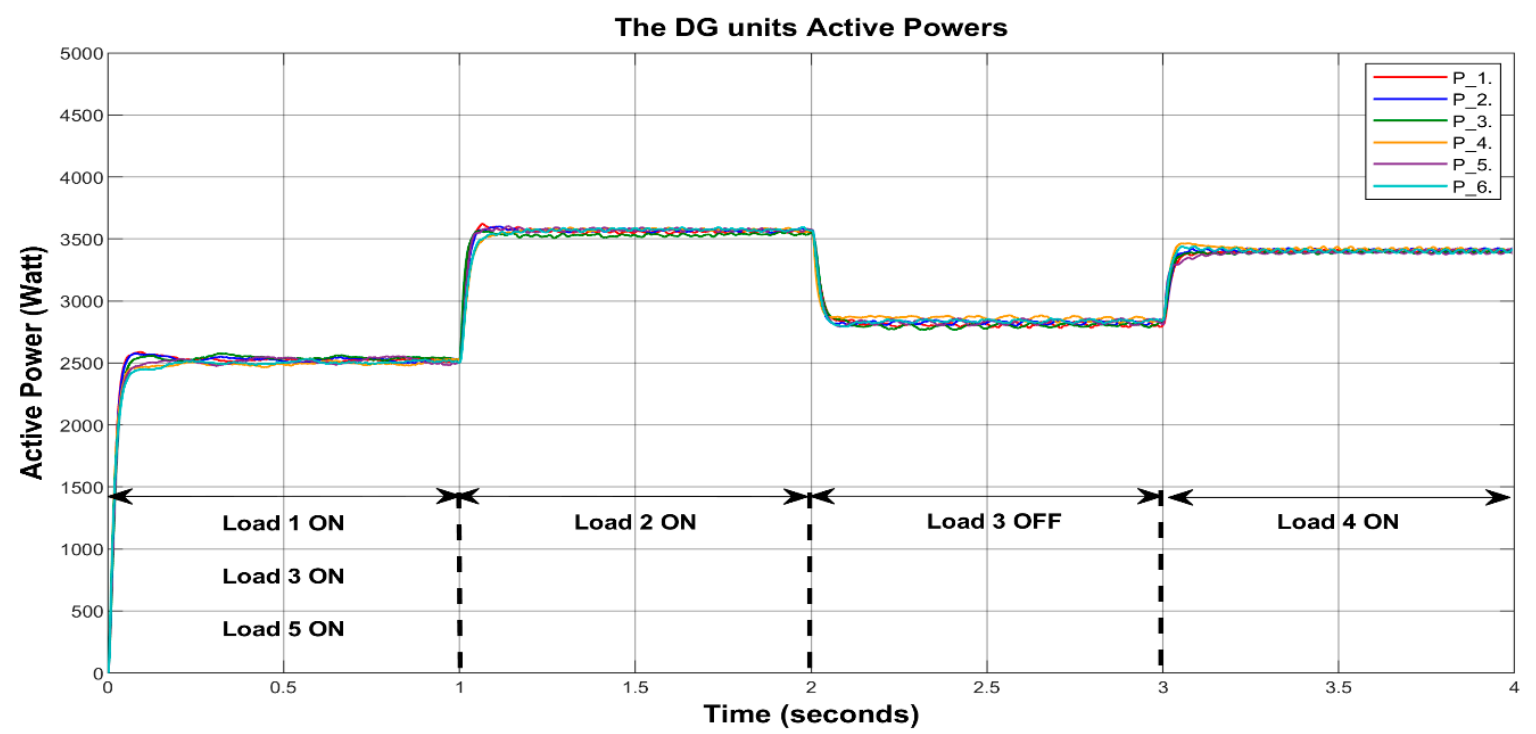

Figure 9. The DG units' active powers for Case 1.

In Figure 9, the active powers shared accurately at $2.5 \mathrm{~kW}$ in $0-1 \mathrm{~s}$ period. In this period, loads 1 and 3, and 5 are connected. Each DG unit supplies these loads by $0.83 \mathrm{~kW}, 0.83 \mathrm{~kW}$, and $0.8 .1 \mathrm{~kW}$, respectively. Hence, the total active power of each DG unit is approximately $2.5 \mathrm{~kW}$. When the load 2 is connected at $1 \mathrm{~s}$ and load 4 is connected at $3 \mathrm{~s}$, the frequencies slightly decrease to increase the active powers in order to meet the power demand, as shown in Figure 9. Dividing the additional active power portions by six DG units, each DG unit should supply $1.15 \mathrm{~kW}$ for connecting load 2 at $1 \mathrm{~s}$ and $0.66 \mathrm{~kW}$ for connecting load 4 at $3 \mathrm{~s}$. Hence, the active powers increase from $2.5 \mathrm{~kW}$ to $3.60 \mathrm{~kW}$ when load 2 is connected at $1 \mathrm{~s}$, and from $2.85 \mathrm{~kW}$ to $3.515 \mathrm{~kW}$ when load 4 switches on. In contrast, the frequencies slightly increase and active powers drop from $3.60 \mathrm{~kW}$ to $2.85 \mathrm{~kW}$ when the load 3 is disconnected at $2 \mathrm{~s}$, as shown in Figures 8 and 9. Thus, the $P / f$ droop control provides accurate active power sharing between the DG units, even though every local primary control works independently.

Figures 10 and 11 present the simulation results of the output voltage magnitudes and reactive power sharing. The $Q / V$ droop control enables the output voltages to accurately regulate the phase-to-ground voltage magnitude at $313 \mathrm{~V}$ in the $0-1 \mathrm{~s}$ period, as shown in Figure 10 . When controlled by primary control alone, the reactive powers of DG units are $2.2 \mathrm{kVar}, 2.15 \mathrm{kVar}, 1.75 \mathrm{kVar}, 2.7 \mathrm{kVar}$, 
$1.8 \mathrm{kVar}$, and $2.65 \mathrm{kVar}$ at $1 \mathrm{~s}$, respectively. Thus, the reactive power sharing provided under this mismatched feeder line condition is poor, as shown in Figure 11. This poor reactive power sharing gets worse under load changing conditions.

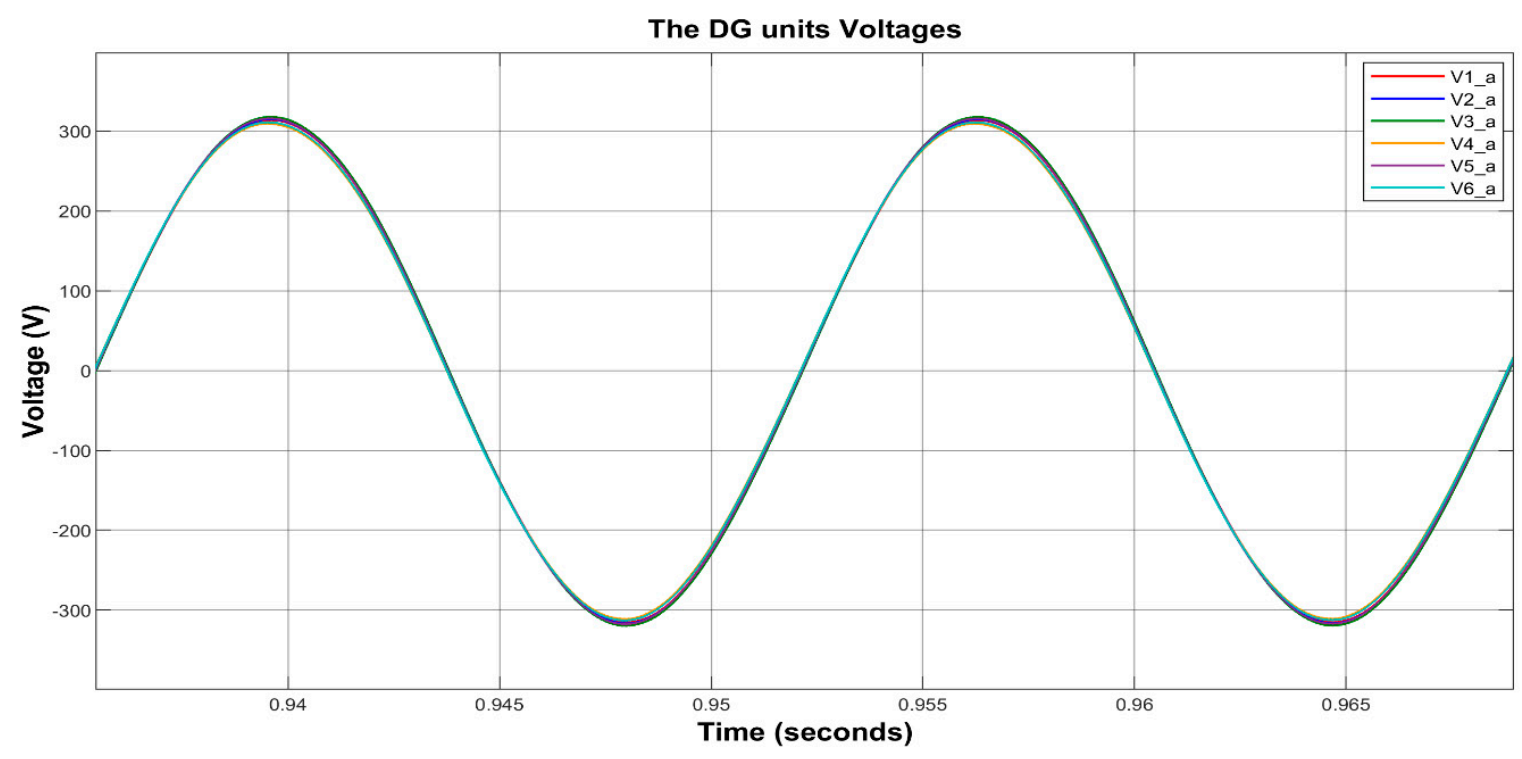

Figure 10. The DG units' magnitude of phase a of output voltages for Case 1.

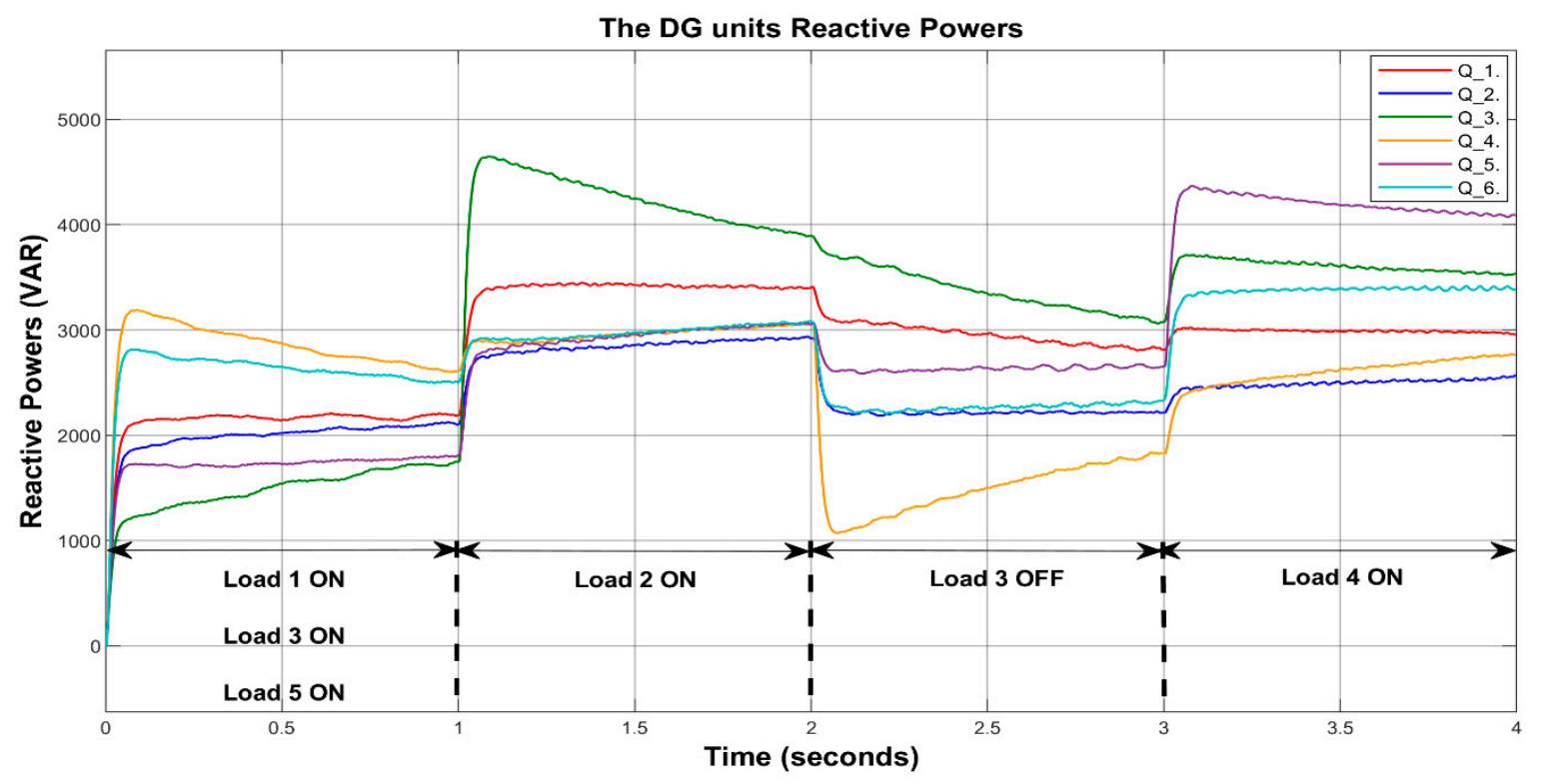

Figure 11. The DG units' reactive powers for Case 1.

The impedance of the feeder lines directly affects the accuracy of the reactive power sharing. Here, the output current magnitude difference between DG 3 and 4 units is about 2A, while the DG output currents in the steady state should be almost the same. Additionally, under sudden load changing the output current difference between DG units increases. Thus, the $Q / V$ droop control fails to regulate the current, as shown in Figure 12 under all load change conditions, and the virtual impedance control with fixed values provides inadequate reactive power sharing under the mismatched feeder line condition. According to IEEE Std 1547.4-2011 [41], a DG unit should be able to provide accurate reactive power to enable the system to effectively meet the load requirements and enhance the stability of the entire islanded microgrid system [41]. The reactive power gain $\left(k_{q i}\right)$ needs to be updated or adjusted based 
on how the mismatched feeder line influences the system based on the reactive power analysis above. A consensus control algorithm is added in the next case to achieve accurate reactive power sharing.

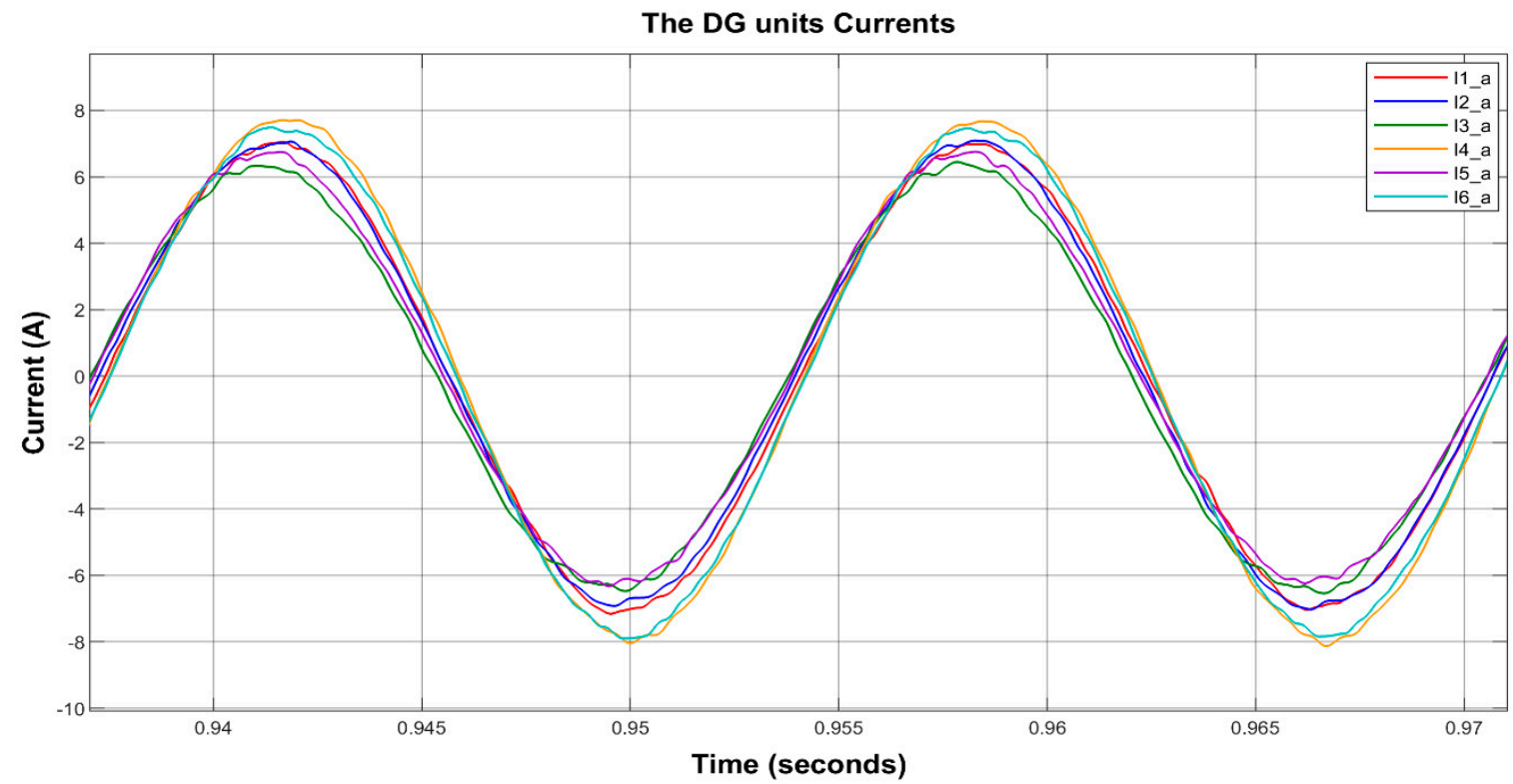

Figure 12. The DG units' of phase a of output current for Case 1.

\subsubsection{Case 2: Leaderless Consensus Control with Ring Communication Topology}

It is clear that the local primary control struggles to share the reactive power accurately. A leaderless consensus control is added to the primary control with a ring communication topology to overcome this challenge. Figures 13 and 14 show that frequency regulation and accurate active power exhibit few artifacts during load change conditions and the results remain almost the same as Case 1. In addition, Figure 15 displays the DG units' output voltages with almost no change when compared to Case 1.

The DG units Frequencies

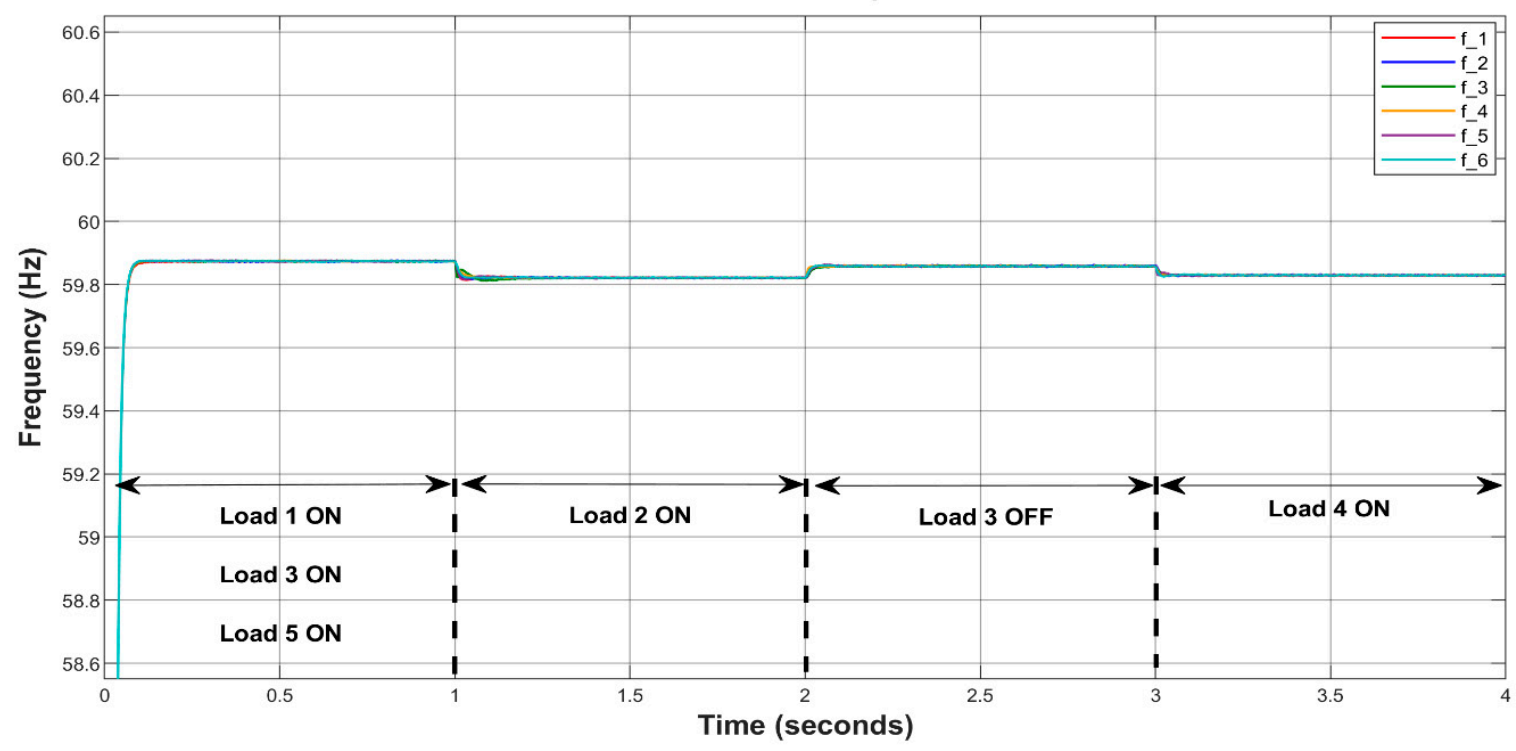

Figure 13. The DG units' frequencies for Case 2. 


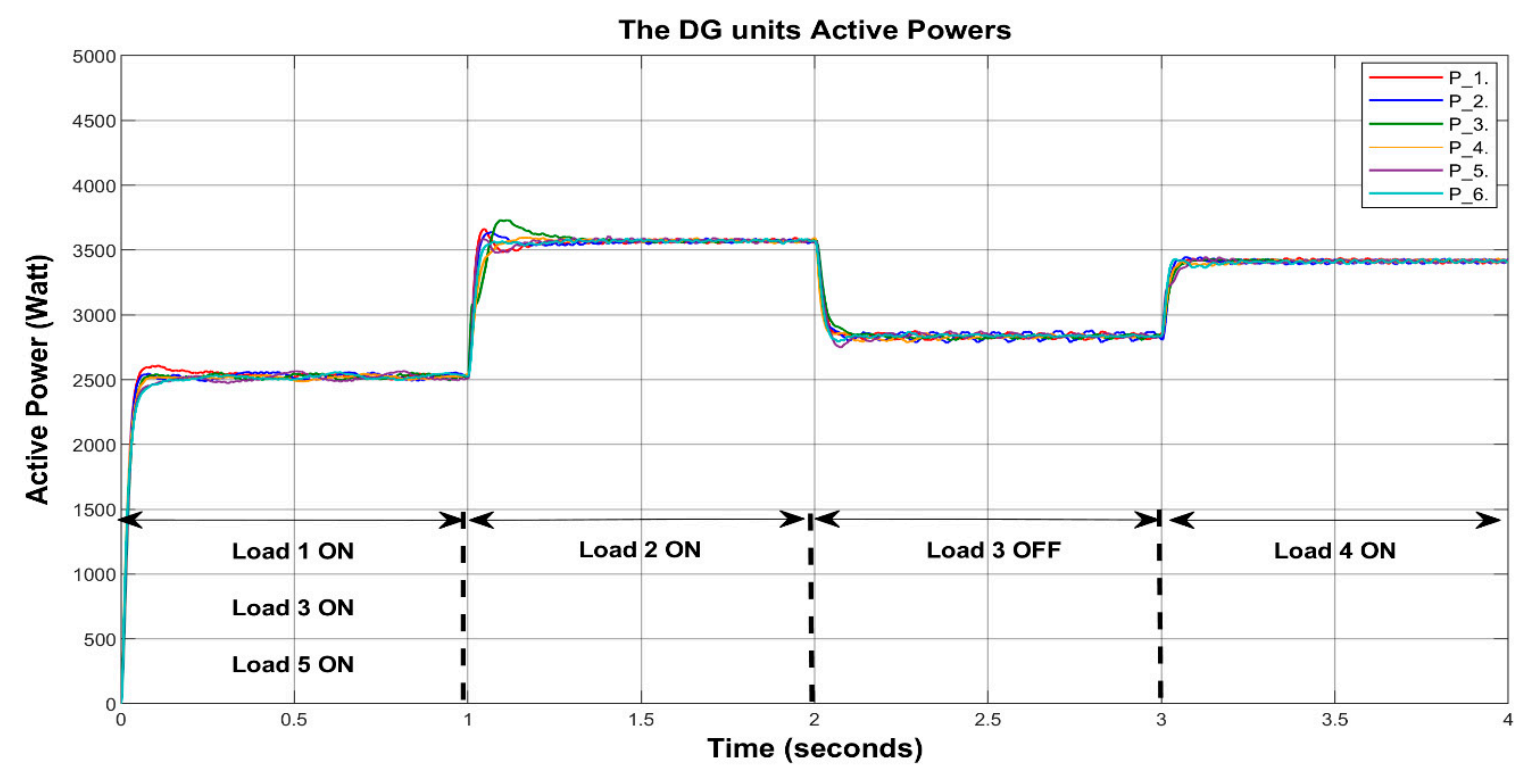

Figure 14. The DG units' active powers for Case 2.

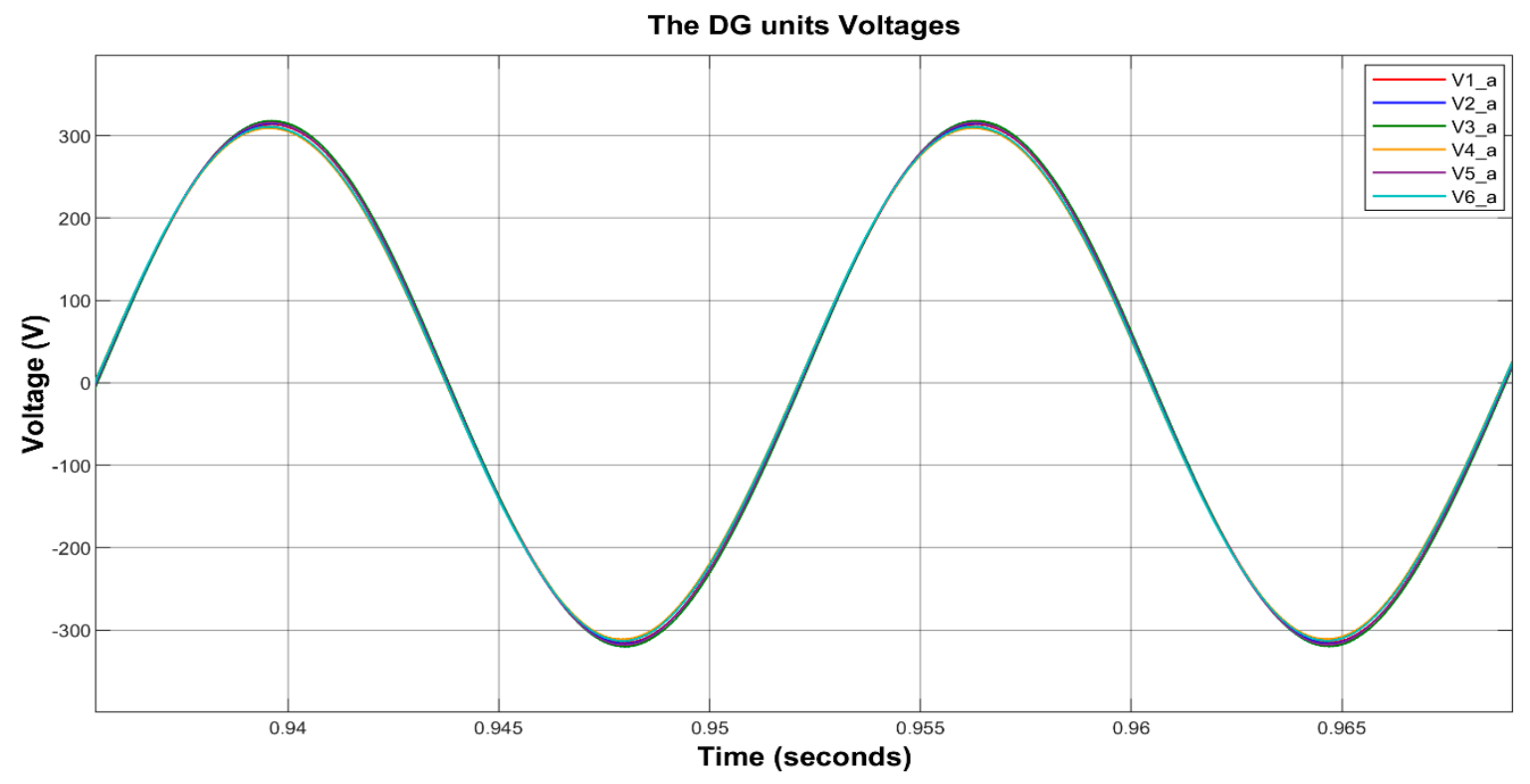

Figure 15. The DG units' magnitude of phase a of output voltages for Case 2.

The difference here is that the leaderless consensus control improves the current regulations, as shown in Figure 16. By comparing Figures 12 and 16 in case 1, it can be seen that the leaderless consensus control successfully removes the current differences that provide an enhancement in reactive power sharing, as in Figure 17. It is obvious that adding consensus control to the proposed control does not impact droop control performance, but solve the poor reactive power challenge, as evident in Figure 17. Here, the consensus control updates the adaptive virtual impedance based on reactive powers of DG unit's neighbors to eliminate the reactive power error. This elimination of reactive power errors improves the current regulation and the virtual impedance ensures an accurate equivalent impedance of the feeder lines. 


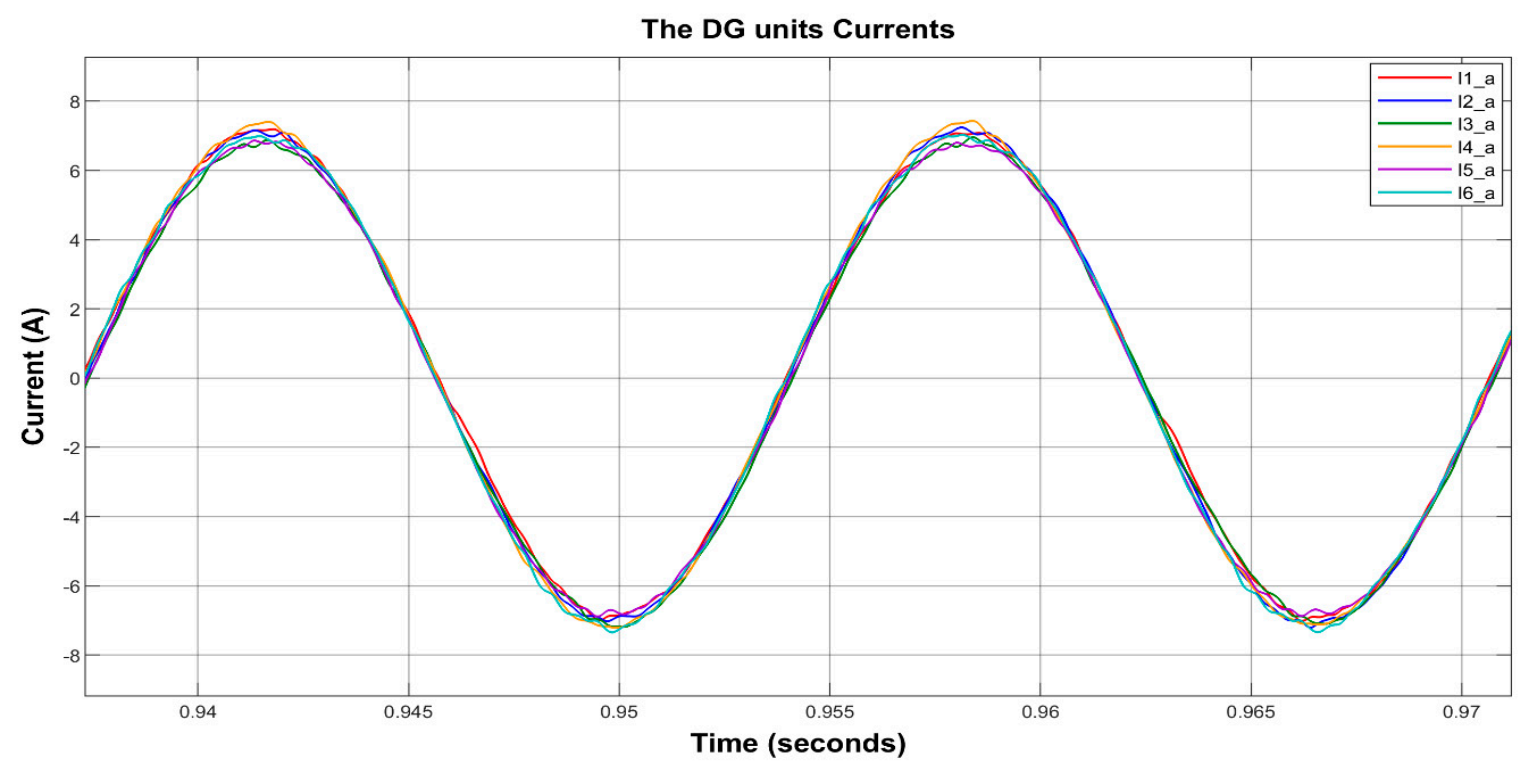

Figure 16. The DG units' output current for Case 2.

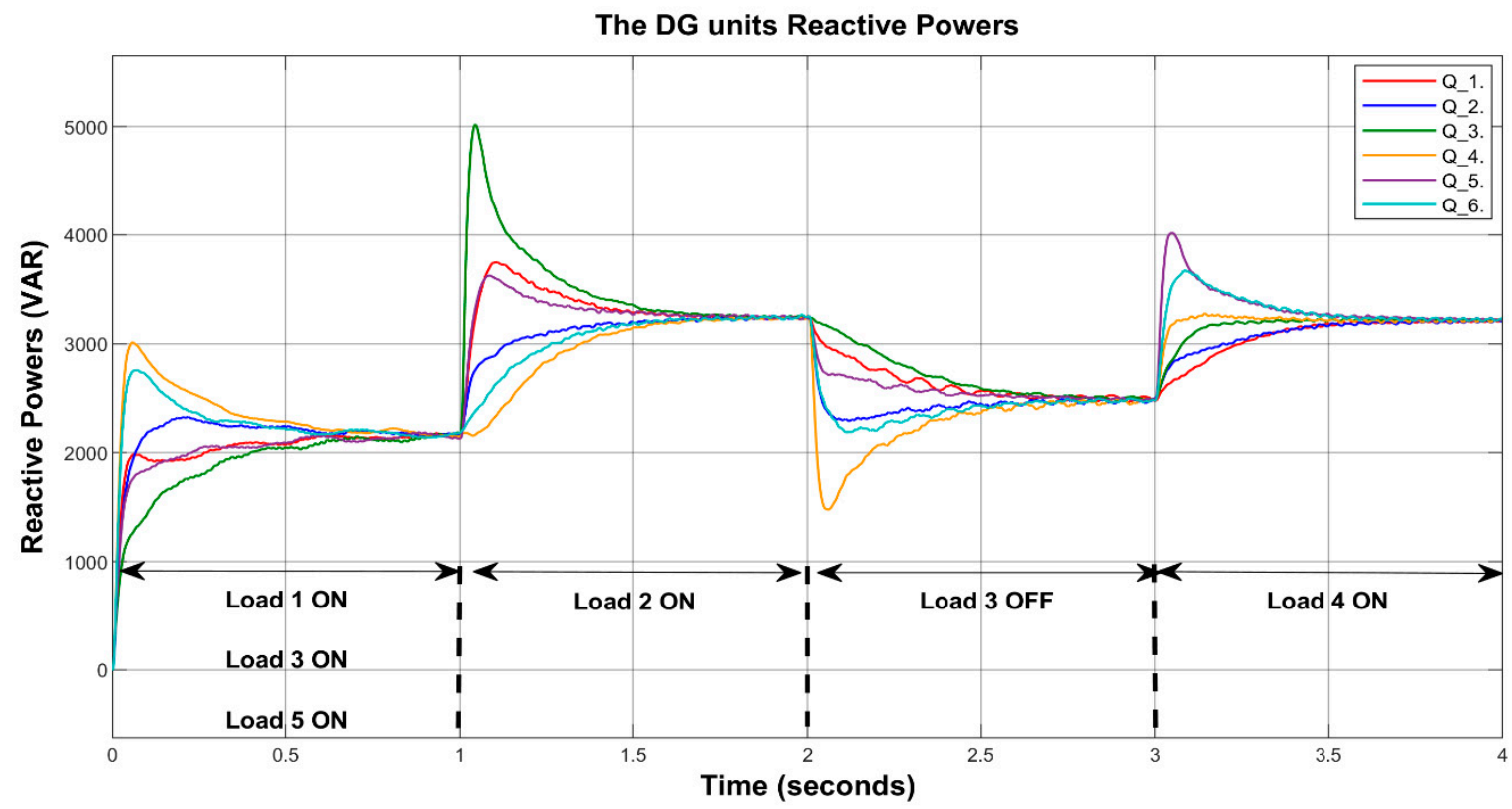

Figure 17. The DG units' reactive powers for Case 2.

Figure 17, below, illustrates reactive power sharing and shows that leaderless consensus control gradually eliminates the reactive power errors between the DG units. When controlled by the proposed method, the reactive powers of DG units converge to a steady state. For example, in the 2-4 s period, each DG unit supplies $0.5 \mathrm{kVar}, 0.81 \mathrm{kVar}, 0.83 \mathrm{kVar}$, and $1.21 \mathrm{kVar}$ for loads 1, 2, 3, and 5, respectively. Hence, the total reactive power that is supplied by each DG unit is $3.35 \mathrm{kVar}$. In this case, the leaderless consensus control takes about $0.75 \mathrm{~s}$ to converge all DG units to the consensus point in all load change conditions, as shown in Figure 17.

According to [19], the rate of convergence of consensus control is quantified by the second smallest eigenvalue $\left(\lambda_{2}\right)$ of the Laplacian matrix, a property that is known as algebraic connectivity [42]. In the undirected communication topology, $\lambda_{2}$ is always greater than zero. The greater the value of $\lambda_{2}$ means that the rate of convergence increases and all DG units reach to the consensus point faster. The Laplacian matrix of the ring communication topology has $\lambda_{2}=1$, given the ring communication topology use in this case. Thus, there is an opportunity to improve the rate of convergence and strengthen the 
connectivity of the communication topology. Here, the failure of any two-communication links can separate the topology into two different parts that brings new challenges to manage this topology in order to control the system. In addition, the failure of one communication link makes exchanging information slower, since the exchange only occurs in one direction and will subsequently influence the rate of convergence.

\subsubsection{Case 3: Leader-Followers Consensus Control with Ring Communication Topology}

Since the reactive power sharing has a slow rate of convergence in the previous case, the leader-followers consensus control algorithm in (13) is simulated with the ring communication topology to accelerate the rate of convergence [34,36]. To implement this control, one of the DG units is selected to act as a leader, while the other DG units follow this leader. Here, DG 2 is selected to lead the other DG units. Applying this approach with a distributed communication topology enhances the rate of convergence, as shown in Figure 18. In Case 2, all DG units require approximately $0.75 \mathrm{~s}$ to converge all DGs' reactive powers to the consensus point, while this consensus control algorithm converges all DG units within $0.40 \mathrm{~s}$. Clearly, the leader-followers consensus control algorithm improves the rate of convergence. However, this consensus control requires extra communication links to make connections between the leader and its followers. In a large system, extra communication links increase the expense of this communication topology and increase the burden and dependence on the leader agent. Therefore, this consensus control topology is not recommended for large systems.

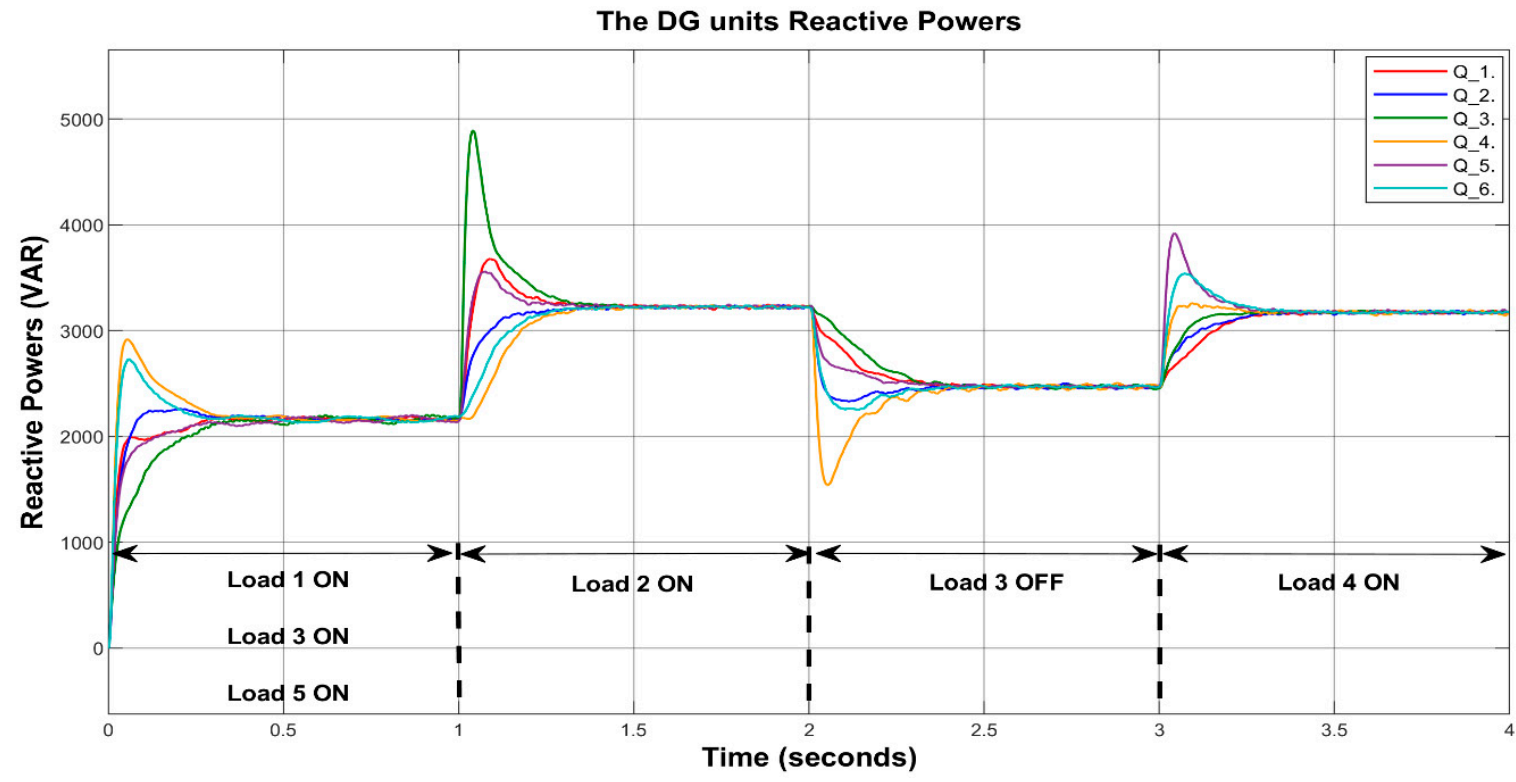

Figure 18. The DG units' reactive powers for Case 3.

\subsubsection{Case 4: Leaderless Consensus Control with Complete Communication Topology}

In Case 4, a complete communication topology, as shown in Figure 4, is simulated in order to find the fastest rate of convergence in this islanded microgrid. Here, every agent behaves as a leader or central DG unit and all DG units share all information to adjust their output values. All DG units achieve consensus within $0.2 \mathrm{~s}$ in all load change conditions, as shown in Figure 19. Even though this complete topology provides the fastest rate of convergence, this topology is impractical for large systems due to its expenses. 
The DG units Reactive Powers

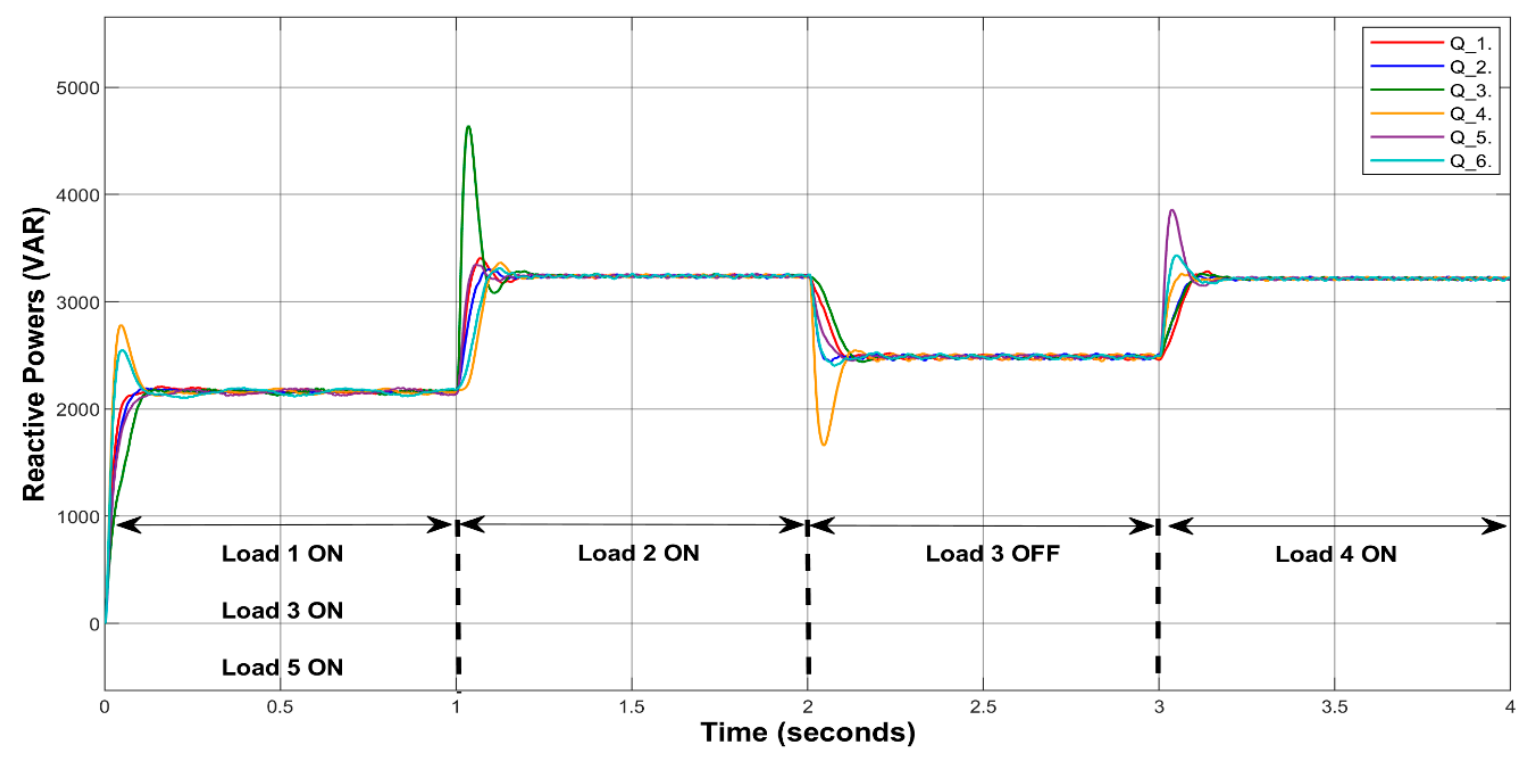

Figure 19. The DG units' reactive powers for Case 4.

\subsubsection{Case 5: Leaderless Consensus Control with Triangle Mesh Communication Topology}

Based on previous cases, there is an inverse relationship between the rate of convergence and the cost of the communication topology. In this case, a triangle mesh communication topology with leaderless consensus control algorithm is proposed in order to find a topology that balances the rate of convergence to consensus and the overall system cost. Figure 5 illustrates the triangle mesh communication topology, where the Laplacian matrix of this communication topology for a 6 DG system can be expressed, as

$$
\mathcal{L}=\left[\begin{array}{rrrrrr}
3 & -1 & -1 & -1 & 0 & 0 \\
-1 & 2 & 0 & -1 & 0 & 0 \\
-1 & 0 & 4 & -1 & -1 & -1 \\
-1 & -1 & -1 & 4 & 0 & -1 \\
0 & 0 & -1 & 0 & 2 & -1 \\
0 & 0 & -1 & -1 & -1 & 3
\end{array}\right]
$$

The Laplacian matrix of the triangle mesh communication topology in (43) has $\lambda_{2}=1.19$, which is greater than $\lambda_{2}=1$ in Case 2. According to [19], a greater value of $\lambda_{2}$ indicates a faster rate of convergence. The triangle mesh communication topology guarantees a faster rate of convergence than the ring topology. Figure 20 shows that all of the DG units converge within $0.45 \mathrm{~s}$. Thus, the triangle mesh communication topology increases the rate of convergence when compared to ring communication topology in Case 2.

Taking the extensibility and scalability of the triangle mesh topology into consideration, this topology can easily expand without shutdown or interruption to the entire system, since adding a new DG unit occurs in the terminal DG unit with two communication links, as shown in Figure 5. The triangle mesh structure takes advantage of the centralized communication concept with some restrictions to reduce the cost and overloading on the central unit(s). Here, each DG unit agent has a maximum limit of four communication links, while the centralized communication topology has no limit. In addition, the DG unit agent that reached the limit of four communication links is located in the center of the topology. Thus, the system can grow organically with a reasonable increase in cost, since this growth does not cause overloading on any one DG unit. 


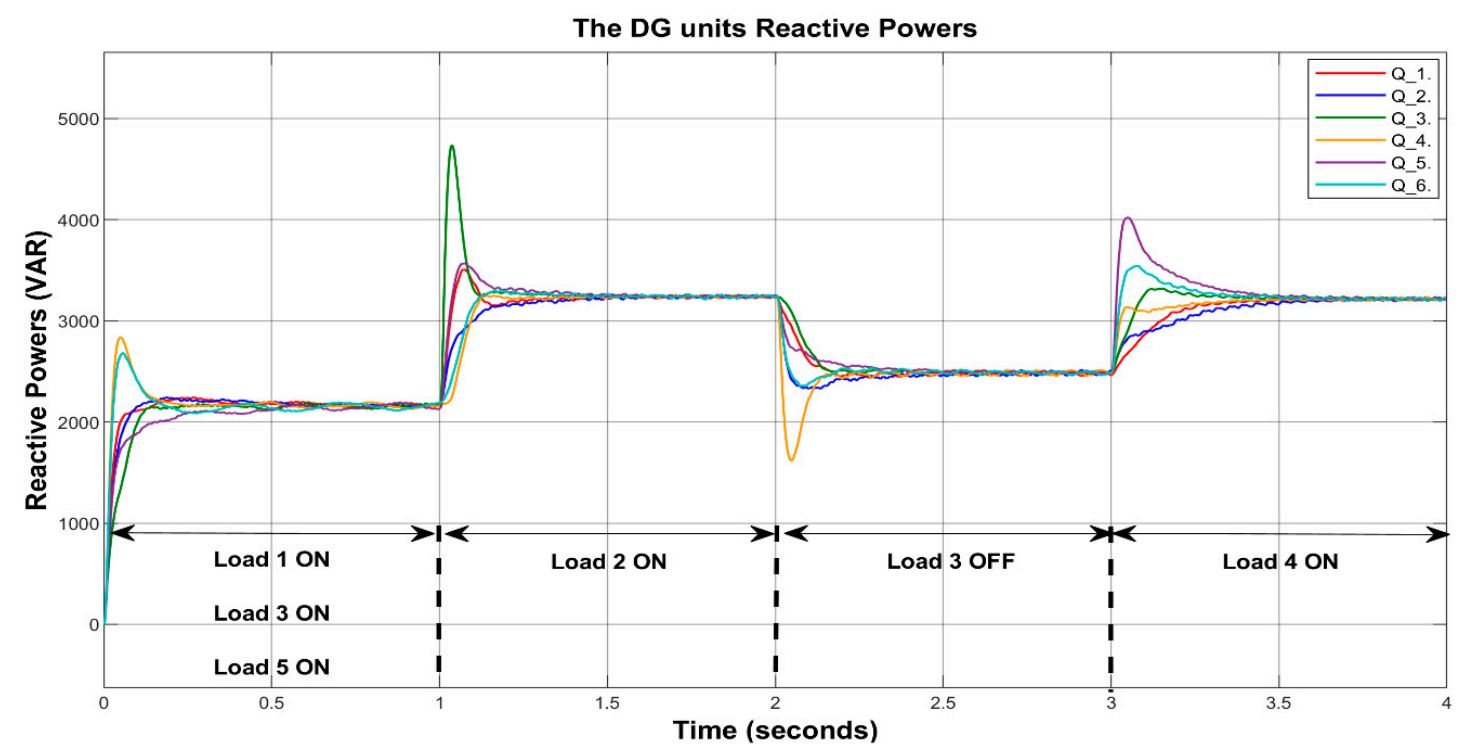

Figure 20. The DG units' reactive powers for Case 5.

\section{Conclusions}

A mathematical model of adaptive virtual impedance control that is based on the leaderless and leader-followers consensus controls with triangle mesh communication topology proposed in this paper eliminates the reactive power sharing errors in a DG microgrid under mismatched feeder line conditions. A MATLAB/Simulink simulation model with six DG units validates the proposed control performance under three different communication topologies, the ring, complete, and triangle mesh. From simulation findings, we conclude that leaderless consensus control algorithm is a reliable option with large systems, while the leader-followers consensus control algorithm is suitable for the small systems, since it requires extra communication links to enable all followers to communicate directly with the leader DG unit agent. The simulation findings show that a triangle mesh communication topology balances between the rate of convergence and the cost of communication topology. In addition, extensibility and scalability are advantages of the triangle mesh topology over the ring and complete topologies. In future works, the fault tolerance of the system and influence of communication time delay will be considered with this proposed control method.

Author Contributions: Conceptualization, A.S.A.; methodology, A.S.A.; software, A.S.A.; validation, A.S.A., and M.W.D.; formal analysis, A.S.A.; investigation, A.S.A.; resources, A.S.A.; data curation, A.S.A.; writing-original draft preparation, A.S.A.; writing-review and editing, A.S.A., and M.W.D.; visualization, A.S.A.; supervision, M.W.D.; Revision, A.S.A., and M.W.D. All authors have contributed to the editing and proofreading of this paper. All authors have read and agreed to the published version of the manuscript.

Funding: This research received no external funding.

Conflicts of Interest: The authors declare no conflict of interest.

\section{References}

1. John, B.; Ghosh, A.; Zare, F. Droop control in low voltage islanded microgrids for sharing nonlinear and unbalanced loads. In Proceedings of the 2017 IEEE Region 10 Symposium (TENSYMP), Cochin, India, 14-16 July 2017; pp. 1-5. [CrossRef]

2. Baojin, L.; Zeng, L.; Jinjun, L.; Teng, W.; Ronghui, A. A novel unbalanced power sharing control method for an islanded microgrid. In Proceedings of the 2017 IEEE 3rd International Future Energy Electronics Conference and ECCE Asia (IFEEC 2017-ECCE Asia), Kaohsiung, Taiwan, 3-7 June 2017; pp. 1617-1622. [CrossRef]

3. Han, H.; Hou, X.; Yang, J.; Wu, J.; Su, M.; Guerrero, J.M. Review of Power Sharing Control Strategies for Islanding Operation of AC Microgrids. IEEE Trans. Smart Grid 2016, 7, 200-215. [CrossRef] 
4. Han, Y.; Li, H.; Shen, P.; Coelho, E.A.A.; Guerrero, J.M. Review of Active and Reactive Power Sharing Strategies in Hierarchical Controlled Microgrids. IEEE Trans. Power Electron. 2017, 32, 2427-2451. [CrossRef]

5. Yao, W.; Chen, M.; Matas, J.; Guerrero, J.M.; Qian, Z.-M. Design and Analysis of the Droop Control Method for Parallel Inverters Considering the Impact of the Complex Impedance on the Power Sharing. IEEE Trans. Ind. Electron. 2011, 58, 576-588. [CrossRef]

6. Guerrero, J.M.; Chandorkar, M.; Lee, T.-L.; Loh, P.C. Advanced Control Architectures for Intelligent Microgrids-Part I: Decentralized and Hierarchical Control. IEEE Trans. Ind. Electron. 2013, 60, 1254-1262. [CrossRef]

7. De Souza, W.F.; Severo-Mendes, M.A.; Lopes, L.A.C. Power sharing control strategies for a three-phase microgrid in different operating condition with droop control and damping factor investigation. IET Renew. Power Gener. 2015, 9, 831-839. [CrossRef]

8. Kim, J.; Guerrero, J.M.; Rodriguez, P.; Teodorescu, R.; Nam, K. Mode Adaptive Droop Control With Virtual Output Impedances for an Inverter-Based Flexible AC Microgrid. IEEE Trans. Power Electron. 2011, 26, 689-701. [CrossRef]

9. Guerrero, J.M.; Berbel, N.; Matas, J.; de Vicuna, L.G.; Miret, J. Decentralized Control for Parallel Operation of Distributed Generation Inverters in Microgrids Using Resistive Output Impedance. In Proceedings of the IECON 2006-32nd Annual Conference on IEEE Industrial Electronics, Paris, France, 6-10 November 2006; pp. 5149-5154. [CrossRef]

10. Shafiee, Q.; Guerrero, J.M.; Vasquez, J.C. Distributed Secondary Control for Islanded Microgrids-A Novel Approach. IEEE Trans. Power Electron. 2014, 29, 1018-1031. [CrossRef]

11. Shafiee, Q.; Stefanović, Č.; Dragičević, T.; Popovski, P.; Vasquez, J.C.; Guerrero, J.M. Robust Networked Control Scheme for Distributed Secondary Control of Islanded Microgrids. IEEE Trans. Ind. Electron. 2014, 61, 5363-5374. [CrossRef]

12. Bidram, A.; Davoudi, A.; Lewis, F.L.; Sam Ge, S. Distributed Adaptive Voltage Control of Inverter-Based Microgrids. IEEE Trans. Energy Convers. 2014, 29, 862-872. [CrossRef]

13. Zhu, Y.; Zhuo, F.; Wang, F.; Liu, B.; Gou, R.; Zhao, Y. A Virtual Impedance Optimization Method for Reactive Power Sharing in Networked Microgrid. IEEE Trans. Power Electron. 2016, 31, 2890-2904. [CrossRef]

14. Kahrobaeian, A.; Ibrahim Mohamed, Y.A.-R. Networked-Based Hybrid Distributed Power Sharing and Control for Islanded Microgrid Systems. IEEE Trans. Power Electron. 2015, 30, 603-617. [CrossRef]

15. Mahmud, M.A.; Hossain, M.J.; Pota, H.R.; Oo, A.M.T. Robust Nonlinear Distributed Controller Design for Active and Reactive Power Sharing in Islanded Microgrids. IEEE Trans. Energy Convers. 2014, 29, 893-903. [CrossRef]

16. Wu, D.; Dragicevic, T.; Vasquez, J.C.; Guerrero, J.M.; Guan, Y. Secondary coordinated control of islanded microgrids based on consensus algorithms. In Proceedings of the 2014 IEEE Energy Conversion Congress and Exposition (ECCE), Pittsburgh, PA, USA, 14-18 September 2014; pp. 4290-4297. [CrossRef]

17. Chen, G.; Guo, Z. Distributed Secondary and Optimal Active Power Sharing Control for Islanded Microgrids With Communication Delays. IEEE Trans. Smart Grid 2019, 10, 2002-2014. [CrossRef]

18. Hoang, T.V.; Lee, H.-H. Distributed Power Sharing Strategy for Islanded Microgrids without Frequency and Voltage Deviations. In Proceedings of the 2018 International Power Electronics Conference (IPEC-Niigata 2018 ECCE Asia), Niigata, Japan, 20-24 May 2018; pp. 1752-1757. [CrossRef]

19. Gulzar, M.M.; Rizvi, S.T.H.; Javed, M.Y.; Munir, U.; Asif, H. Multi-Agent Cooperative Control Consensus: A Comparative Review. Electronics 2018, 7, 22. [CrossRef]

20. Ji, M.; Egerstedt, M. Distributed Coordination Control of Multiagent Systems While Preserving Connectedness. IEEE Trans. Robot. 2007, 23, 693-703. [CrossRef]

21. Bisht, N.; Singh, S. analytical study of different network topologies. Int. Res. J. Eng. Technol. (IRJET) 2015, 2, $88-90$.

22. Kar, S.; Moura, J.M.F. Topology for Global Average Consensus. In Proceedings of the 2006 Fortieth Asilomar Conference on Signals, Systems and Computers, Pacific Grove, CA, USA, 29 October-1 November 2006; pp. 276-280. [CrossRef]

23. Olfati-Saber, R.; Murray, R.M. Consensus problems in networks of agents with switching topology and time-delays. IEEE Trans. Autom. Control 2004, 49, 1520-1533. [CrossRef]

24. Belykh, I.; Hasler, M.; Lauret, M.; Nijmeijer, H. Synchronization and graph topology. Int. J. Bifurc. Chaos 2005, 15, 3423-3433. [CrossRef] 
25. Zhang, H.; Kim, S.; Sun, Q.; Zhou, J. Distributed Adaptive Virtual Impedance Control for Accurate Reactive Power Sharing Based on Consensus Control in Microgrids. IEEE Trans. Smart Grid 2017, 8, 1749-1761. [CrossRef]

26. Azim, M.I.; Hossain, M.J.; Griffith, F.H.M.R.; Pota, H.R. An improved droop control scheme for islanded microgrids. In Proceedings of the 2015 5th Australian Control Conference (AUCC), Gold Coast, QLD, Australia, 5-6 November 2015; pp. 225-229.

27. Wu, Y.; Guerrero, J.M.; Wu, Y. Distributed coordination control for suppressing circulating current in parallel inverters of islanded microgrid. Transm. Distrib. IET Gener. 2019, 13, 968-975. [CrossRef]

28. Wang, Y.; Nguyen, T.L.; Xu, Y.; Li, Z.; Tran, Q.-T.; Caire, R. Cyber-Physical Design and Implementation of Distributed Event-Triggered Secondary Control in Islanded Microgrids. IEEE Trans. Ind. Appl. 2019, 55, 5631-5642. [CrossRef]

29. Lu, X.; Yu, X.; Lai, J.; Wang, Y.; Guerrero, J.M. A Novel Distributed Secondary Coordination Control Approach for Islanded Microgrids. IEEE Trans. Smart Grid 2018, 9, 2726-2740. [CrossRef]

30. Yan, Y.; Shi, D.; Bian, D.; Huang, B.; Yi, Z.; Wang, Z. Small-Signal Stability Analysis and Performance Evaluation of Microgrids Under Distributed Control. IEEE Trans. Smart Grid 2019, 10, 4848-4858. [CrossRef]

31. ChethanRaj, D.; Gaonkar, D.N. Multiple Inverters Operated in Parallel for Proportional Load Sharing in Microgrid. Int. J. Power Electron. Drive Syst. 2017, 8, 654. [CrossRef]

32. Van Steen, M. Graph Theory and Complex Networks. Introduction 2010, 144, 287.

33. Bondy, J.A.; Murty, U.S.R. Graph Theory with Applications; Macmillan: London, UK, 1976; Volume 290. [CrossRef]

34. Khayat, Y.; Shafiee, Q.; Heydari, R.; Naderi, M.; Dragicevic, T.; Simpson-Porco, J.W.; Dorfler, F.; Fathi, M.; Blaabjerg, F.; Guerrero, J.M.; et al. On the Secondary Control Architectures of AC Microgrids: An Overview. IEEE Trans. Power Electron. 2019. [CrossRef]

35. Olfati-Saber, R.; Fax, J.A.; Murray, R.M. Consensus and Cooperation in Networked Multi-Agent Systems. Proc. IEEE 2007, 95, 215-233. [CrossRef]

36. Ortega, G.; Muñoz, F.; Quesada, E.S.E.; Garcia, L.R.; Ordaz, P. Implementation of leader-follower linear consensus algorithm for coordination of multiple aircrafts. In Proceedings of the 2015 Workshop on Research, Education and Development of Unmanned Aerial Systems (RED-UAS), Cancun, Mexico, 23-25 November 2015; pp. 25-32. [CrossRef]

37. Xu, Y.; Sun, H.; Gu, W.; Xu, Y.; Li, Z. Optimal Distributed Control for Secondary Frequency and Voltage Regulation in an Islanded Microgrid. IEEE Trans. Ind. Inform. 2019, 15, 225-235. [CrossRef]

38. Lewis, F.L.; Zhang, H.; Hengster-Movric, K.; Das, A. Cooperative Control of Multi-Agent Systems: Optimal and Adaptive Design Approaches; Communications and Control Engineering; Springer: London, UK, 2014; ISBN 978-1-4471-5573-7. [CrossRef]

39. Godsil, C.; Royle, G.F. Algebraic Graph Theory; Graduate Texts in Mathematics; Springer: New York, NY, USA, 2001; ISBN 978-0-387-95241-3. [CrossRef]

40. Khalil, H.K. Nonlinear Systems; Prentice hall: Upper Saddle River, NJ, USA, 2002.

41. IEEE Guide for Design, Operation, and Integration of Distributed Resource Island Systems with Electric Power Systems. IEEE Std. 2011, 1-54. [CrossRef]

42. Fiedler, M. Algebraic connectivity of graphs. Czechoslov. Math. J. 1973, 23, 298-305.

(C) 2020 by the authors. Licensee MDPI, Basel, Switzerland. This article is an open access article distributed under the terms and conditions of the Creative Commons Attribution (CC BY) license (http://creativecommons.org/licenses/by/4.0/). 